\title{
On the role of secondary pions in spallation targets
}

\author{
Davide Mancusi ${ }^{1 a}$, Sergio Lo $\mathrm{Meo}^{23}$, Nicola Colonna ${ }^{4}$, Alain Boudard ${ }^{5}$, Miguel Antonio Cortés-Giraldo ${ }^{6}$, Joseph \\ Cugnon $^{7}$, Jean-Christophe David ${ }^{5}$, Sylvie Leray ${ }^{5}$, Jorge Lerendegui-Marco ${ }^{6}$, Cristian Massimi ${ }^{38}$, and Vasilis \\ Vlachoudis ${ }^{9}$ \\ 1 Den-Service d'étude des réacteurs et de mathématiques appliquées (SERMA), CEA, Université Paris-Saclay, F-91191, Gif- \\ sur-Yvette, France \\ 2 ENEA, Research Centre "Ezio Clementel", I-40129 Bologna, Italy \\ 3 INFN, Section of Bologna, I-40127 Bologna, Italy \\ 4 INFN, Section of Bari, I-70125 Bari, Italy \\ 5 IRFU, CEA, Université Paris-Saclay, F-91191, Gif-sur-Yvette, France \\ 6 Universidad de Sevilla, Facultad de Fisica, 41012 Sevilla, Spain \\ 7 AGO department, University of Liège, allée du 6 août 17, bât. B5, B-4000 Liège 1, Belgium \\ 8 Physics and Astronomy Dept. "Alma Mater Studiorum" - University of Bologna, I-40126 Bologna, Italy \\ 9 European Organization for Nuclear Research (CERN), CH-1211 Geneva, Switzerland
}

Received: December 19, 2016

\begin{abstract}
We use particle-transport simulations to show that secondary pions play a crucial role for the development of the hadronic cascade and therefore for the production of neutrons and photons from thick spallation targets. In particular, for the $\mathrm{n}_{-} \mathrm{TOF}$ lead spallation target, irradiated with $20 \mathrm{GeV} / c$ protons, neutral pions are involved in the production of $\sim 90 \%$ of the high-energy photons; charged pions participate in $\sim 40 \%$ of the integral neutron yield. Nevertheless, photon and neutron yields are shown to be relatively insensitive to large changes of the average pion multiplicity in the individual spallation reactions. We characterize this robustness as a peculiar property of hadronic cascades in thick targets.
\end{abstract}

PACS. 25.40.Sc Spallation reactions - 24.10.Lx Monte-Carlo simulations - 28.20.Gd Neutron transport: diffusion and moderation

\section{Introduction}

In spallation reactions, a high-energy $(>150 \mathrm{MeV})$ light projectile collides with a nucleus and on average leads to the emission of a large number of particles, mostly neutrons. The spectrum of spallation neutrons extends to large energies, up to the energy of the incoming projectile. For this reason, spallation reactions are often used for the purpose of generating intense high-energy neutron fluxes [1, as it is the case for instance in Accelerator-Driven Systems (ADS), subcritical reactor cores that are kept in a steady state by neutrons produced by a spallation source [2].

Neutrons are not the only particles that are emitted during spallation reactions. Protons and light charged particles (LCPs, $A \leq 4$ ) are also present, as are pions if the projectile energy is high enough. Spallation is actually capable of producing (with varying yields) all nuclei lighter than the target nucleus and close to the stability valley, as well as a handful of nuclei heavier than the target nucleus (as amply demonstrated by several experi-

\begin{tabular}{lll}
\hline a Corresponding author. E-mail address: da- \\
vide.mancusi@cea.fr
\end{tabular}

mental campaigns [see e.g. 3. Fig. 12]). All these particles, especially the lightest ones (neutrons, protons, pions and LCPs), are capable of inducing secondary nuclear reactions in a thick spallation target, and may thus contribute to the development of the hadronic cascade, to particle emission and to the production of residual nuclei [see e.g. 4.

The standard theoretical tool for the description of spallation reactions is a hybrid nuclear-reaction model where an intranuclear-cascade (INC) stage is followed by an optional pre-equilibrium stage and by a statistical de-excitation stage [1]. For the reasons evoked in the previous paragraph, these models must be validated not only for the primary reactions (typically reaction between fast protons and heavy nuclei such as tungsten, lead or bismuth), but also for all secondary reactions that may sizably contribute to the production of neutrons or to any other observable one may be interested in. It is generally acknowledged that secondary proton- and neutron-nucleus reactions are important, as suggested by the selection of validation data for international nuclear-reaction-model intercomparisons [5] 7]; however, the same intercomparisons devoted little attention to the production of secondary pions and to the 
validation of models on pion-induced reactions. This is at least partly due to the fact that inclusive data for pionnucleus reactions are scarce, and partly to the fact that ADSs are expected to operate at energies of the order of $1 \mathrm{GeV}$ [2], where pion multiplicities are relatively low.

Several spallation neutron sources are currently operational around the world and more are under construction or planned for the near future. Predictions of the neutronsource characteristics can typically be obtained by means of Monte Carlo (MC) simulations. Reliable results require detailed and accurate knowledge of the physical processes at the basis of the spallation reactions. Among the currently operating spallation neutron sources, the $n$ TOF (neutron Time-Of-Flight) facility [8] is an intense pulsed neutron source located at CERN. Neutrons are produced by spallation of lead nuclei caused by an incident $20 \mathrm{GeV} / c$ proton beam, and subsequently moderated and collimated towards two experimental areas, where their energy can be measured using the time-of-flight technique. One of the foremost advantages of the detection capability of $\mathrm{n}_{-}$TOF is that the produced neutrons extend over more than twelve orders of magnitude, from thermal energies to the GeV range, allowing highly accurate measurements for a wide range of applications. Precise characterization of the neutron source is crucial for these purposes, and some features of the neutron beam must be inevitably determined via numerical simulations [9]. In recent publications [10, 11], the Geant4 toolkit for particle transport [12, 13. was used to characterize the neutron and photon fluxes directed towards the $\mathrm{n}_{-}$TOF experimental areas. Calculations of neutron and photon fluences performed with different Geant4 physics lists exhibited large relative differences. The authors suggested at the time that this difference could be related to different treatments of pion production and pion-induced reactions.

In this work, we study the role of pion production and its influence on the spallation yields. In particular, it will be shown that secondary pions play a crucial role for particle production in thick spallation targets, such as the $n$ TOF neutron source. We shall demonstrate that the production of high-energy prompt photons is essentially dominated by $\pi^{0}$ decay; this phenomenon is well known in the context of the phenomenology of calorimetric measurements for high-energy physics [14]. At the same time, the production of neutrons is affected by both secondary $\pi^{ \pm}$nucleus reactions and $\pi^{0}$ production. These facts notwithstanding, particle yields are less sensitive to the detail of the specific nuclear-reaction model used for the particletransport simulation. This is explained in terms of an intrinsic "resilience" of hadronic cascades in thick targets.

The paper is structured as follows. In Sec. 2 we provide a brief description of the salient features of the Liège Intranuclear-Cascade model (INCL), which is pivotal for our numerical simulations of the $\mathrm{n}_{-}$TOF spallation target. Thin-target model calculations related to the pion sector are presented and discussed in Sec. 3, along with comparisons against experimental data. Section 4 shifts the focus towards the thick-target transport calculations of the $\mathrm{n}_{-}$TOF spallation target. The most important fea- tures of the MC simulations, described in detail in recent papers [10, 11], are recalled in Secs. 4.1 and 4.2. The role played by pions in the emission of neutrons and photons is highlighted in Secs. 4.3 4.5. Section 4.7 illustrates the tendency of the hadronic cascade to mitigate the sensitivity of the particle yields to the details of the description of the nuclear reactions. Conclusions are drawn in Sec. 5.

\section{Model description: the Liège Intranuclear Cascade model}

The Liège Intranuclear Cascade model (INCL) [15, 16] is one of the most refined existing tools for the description of spallation reactions. The model is currently maintained and developed jointly by the University of Liège (Belgium) and CEA (Saclay, France). The model assumes that the first stage of the reaction can be described as an avalanche of independent binary collisions. The INCL model is essentially classical, with the addition of a few suitable ingredients that mimic genuine quantum-mechanical features of the initial conditions and of the dynamics: for instance, target nucleons are endowed with Fermi motion, realistic space densities are used, the output of binary collisions is random and elementary nucleon-nucleon collisions are subject to Pauli blocking. The model can describe the emission of nucleons and pions; light clusters (up to $Z=5$, $A=8$ by default) can also be produced through a dynamical phase-space coalescence algorithm.

Intranuclear-cascade models in general (and INCL in particular) only describe the fast, dynamical stage of a spallation reaction, leading to the formation of excited nuclei which subsequently de-excite by emitting particles and/or fissioning. It is therefore necessary to follow the de-excitation of this cascade remnant if one requires a complete description of the nuclear reaction. Since the time scale for de-excitation is much longer than for cascade, a different physical description is usually employed. This may include an optional pre-equilibrium stage, which then handles the thermalization of the remnant; if preequilibrium is used, the intranuclear-cascade stage is stopped earlier. Either way, thermalization is attained and subsequent de-excitation of the remnant is described by statistical de-excitation models. Within Geant4, INCL can be directly coupled with two different de-excitation codes, namely: G4ExcitationHandler, the native statistical deexcitation model of Geant4 and the default choice [17, and ABLA V3, a de-excitation model developed at GSI (Darmstadt, Germany) [18, 19]. We stress here that this is not the code that is usually coupled to INCL (which is ABLA07 20]), but rather an older version. A detailed comparison of the capabilities of the two versions can be found in Ref. 20.

Different particles are produced in different stages of the spallation reactions. In particular, while neutrons and $\gamma$-rays are mostly generated in de-excitation processes, pion production, in particular, occurs entirely in the first reaction stage. The pion dynamics in INCL has been recently upgraded to push the upper energy limit of the 
model up to $15-20 \mathrm{GeV}$. Older versions of INCL considered only one mechanism for pion production, namely excitation and subsequent decay of the $\Delta(1232)$ resonance. For nucleon-induced reactions, this is a good approximation up to energies of about $2-3 \mathrm{GeV}$. This is proven by the results of the IAEA benchmark [5]7], as well by the previous studies on the INCL pion dynamics [21, 22]. Additionally, one should not forget that, as soon as multiple collisions are involved, any particle correlation due to the action of an intermediate resonance will be washed out. For the purpose of correctly describing multiple-collision reactions, it is sufficient to capture the first-order behavior, and correlations may be neglected. Of course, selective or exclusive observables (such as two-particle correlations), especially if related to one- or few-collision reactions will generally be incorrectly described.

Above $2-3 \mathrm{GeV}$, excitation of heavier baryonic and mesonic resonances becomes likely 1 . A straightforward extension of INC would in principle entail the description of all the energy-angle-differential cross sections for the formation, scattering and absorption of the resonances in the nuclear medium, as well as their mean-field potentials, decay modes, etc. The amount of information that must be fed into the model is ponderous; besides, most of the time, the available experimental information on these elementary processes is direly scarce, or partial at best. One possible approach would be to rely on an independent event generator for the elementary hadron-nucleus collisions, in the spirit of Ref. 23. In this paper, however, we explore a different solution.

It should be noted that baryonic resonances above $\Delta(1232)$ are largely overlapping. This raises the question of whether it is meaningful to consider them as having separate identities in the framework of INC. Additionally, their lifetime (in vacuum) is much smaller than the typical time between subsequent collisions during INC (a few $\mathrm{fm} / c$ ), so that a heavy resonance is unlikely to undergo any collision before decaying in the nucleus. This is already marginally the case for $\Delta(1232)$, whose lifetime in vacuum is $\sim 1.6 \mathrm{fm} / c$, and indeed most of the observables calculated in INC are rather insensitive to variations of the $\Delta(1232)$ lifetime. It should also be considered that the final (on the time scale of INC) decay products of baryonic resonances are often pions.

Strictly speaking, the arguments above do not apply to most of the lightest unflavored mesonic resonances $(\eta$, $\left.\omega, \eta^{\prime} \ldots\right)$, whose lifetimes are comparable to or longer than the duration of the INC stage; nor do they apply to strange baryons and mesons $(\Lambda, \Sigma, K \ldots)$, which undergo weak decay. However, the available experimental elementary cross sections associated with the production of these particles [24, 25] and order-of-magnitude estimates suggest that their global influence on the INC dynamics

\footnotetext{
1 The excitation of the Roper $N^{*}(1440)$ resonance is a special exception, because it may be excited at lower energy in the $T=0$ channel. INCL4.6 assumes that the kinematics of pion production in this channel is governed by the $\Delta(1232)$ resonance. The extended version of INCL does not make this approximation.
}

is weak. Therefore, it should be possible to treat them as corrections, at least in the energy range up to $10-15 \mathrm{GeV}$.

In view of the discussion above, it is appropriate to use a more pragmatic approach to the description of highenergy reactions. In the latest version of the INCL model, the production and decay of individual resonances (except for $\Delta(1232))$ is bypassed and replaced by multipion collisions, i.e. effective two-body collisions leading to the production of one or more pions in the final state, of the following form:

$$
\begin{aligned}
N+N & \rightarrow N+N+x \pi, \\
\pi+N & \rightarrow N+x \pi .
\end{aligned}
$$

In the current model, the number $x$ of pions in the final state of the collision takes all values from 1 to 4 inclusive.

The rest of the pion dynamics in the new version of INCL is the same as in the older one. The formation, absorption and decay of the $\Delta(1232)$ resonance is explicitly treated. Pion absorption is possible only via the formation of $\Delta(1232)$. No one-step mechanism for pion absorption on nucleon pairs is included. Further details on the latest version of INCL can be found in Refs. 26, 27, Relative to the published version, the current implementation of the model has slightly evolved, with the most notable difference concerning the biasing of nucleons towards the forward direction in the center-of-mass system ${ }^{2}$

The effect of the multipion extension can be studied by comparing some global quantities calculated with the old (INCL4.6) and the new extended model (INCL ++ ). Figure 1 shows the average pion multiplicity (i.e. the average number of pions produced per inelastic reaction) in $\mathrm{p}+{ }^{208} \mathrm{~Pb}$ as a function of the proton energy. While the two models yield similar predictions at low projectile energy, in the older model the multiplicity saturates around $5 \mathrm{GeV}$, never exceeding $\sim 1$ pion per reaction, while the extended model yields an almost linear increase up to $20 \mathrm{GeV}$. Figure 2 shows how the produced pions are distributed over the three charge states, according to the calculations of the extended model. The fraction of neutral pions is roughly energy-independent. On the contrary, the lines for positive and negative pions cross between 4 and $5 \mathrm{GeV}$. The suppression of negative pions at low energy can be explained by considering that the projectile (a proton) carries positive isospin, and that pions can only be produced in the first few collisions. As the energy and the number of collisions increase, pion production becomes increasingly dominated by the total isospin of the system, which is

\footnotetext{
${ }^{2}$ In the current model, the final-state particle momenta are generated according to a flat, unbiased phase-space sampling algorithm. Let $E$ be the generated CM energy of the first nucleon; the value of $E$ determines the minimum $\left(t_{\min }\right)$ and maximum $\left(t_{\max }\right)$ values of the Mandelstam four-momentum transfer $t$. The value of $t$ is then sampled from a distribution of the form $\exp (B t)$ and all the generated momenta are rotated to match the sampled four-momentum transfer for the first nucleon. Clearly this algorithm does not modify the singleparticle energy distributions in the CM system, which are therefore still given by the phase-space model. On the other hand, the distributions in the laboratory system are different.
} 


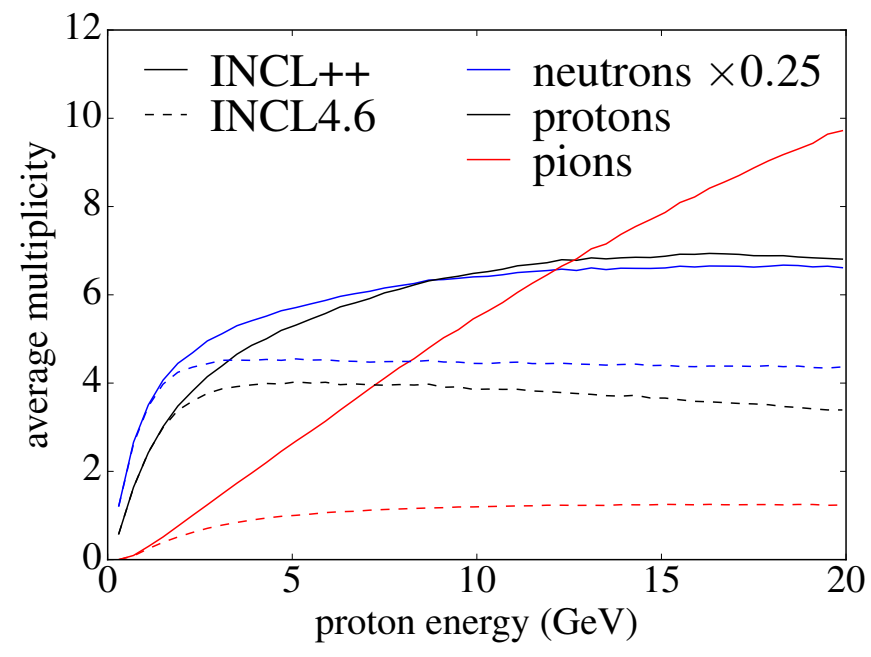

Figure 1. Excitation function for the total average neutron proton and pion multiplicities (from intranuclear cascade and de-excitation) in the final state of $\mathrm{p}+{ }^{208} \mathrm{~Pb}$ reactions, as calculated with (INCL ++ ) and without (INCL4.6) multipion extension, coupled with ABLA07. Note that the neutron curve has been renormalized by a factor of 0.25 .

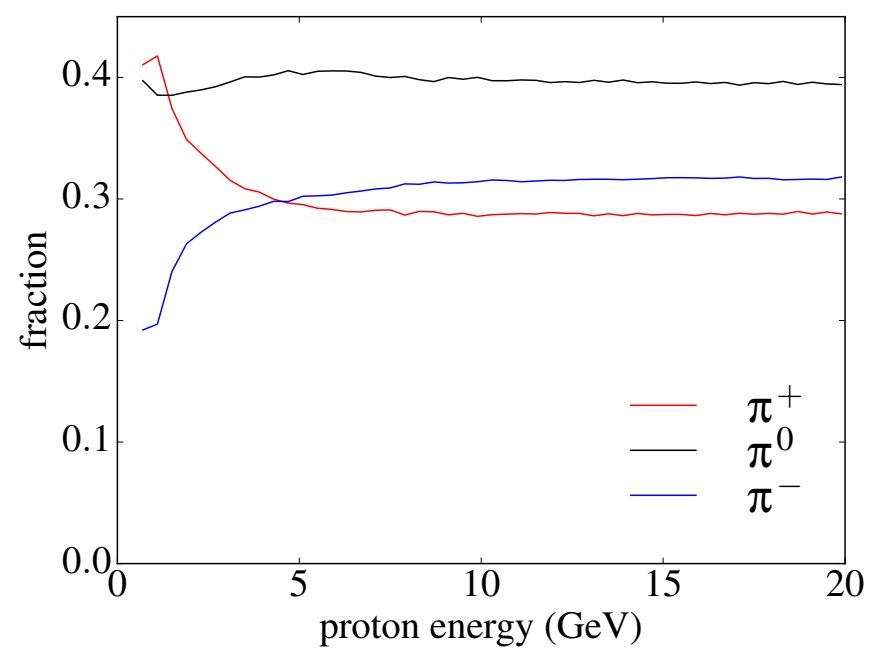

Figure 2. Excitation functions for the average fraction of produced pions for each of the three charge states, in $\mathrm{p}+{ }^{208} \mathrm{~Pb}$, as calculated by INCL ++ .

negative because $N>Z$ in lead. Therefore, negative pions are asymptotically more abundantly produced than positive pions.

For completeness, we mention that the version of the INCL model that was used for the present work is INCL ++ v5.2.9.2.

\section{Thin target: pion-production cross sections}

As discussed in Sec. 1, Geant4 simulations performed with an INCL ++ -based physics list yield the best overall reproduction of the measured neutron production for the $\mathrm{n}$ TOF spallation target, contrary to the physics lists using the Binary Cascade (BIC) [28] or Bertini models [29], which overestimate the experimentally evaluated neutron production by as much as 70\% [10. In Ref.10]it was hinted that a possible explanation of this difference could be related to pion production. In particular, it was pointed out that both neutral and charged pions could play an important role in determining the production of neutrons as well as of the so-called prompt $\gamma$-ray component, i.e. those produced in the first nanoseconds of the spallation reactions (with the delayed $\gamma$-ray component produced later on from neutron capture reaction and de-excitation of excited residues). In the following, the role of secondary pions in spallation targets is investigated, starting from a comparison of theoretical differential cross sections with the available experimental data. We remark that the predictive capability of the INCL model for the production of other particles (in particular neutrons, protons and light charged particles) below $3 \mathrm{GeV}$ has already been established in an extensive benchmark of spallation models, organized under the auspices of the IAEA [5-7].

In order to assess the validity of the INCL ++ and other models, it is very useful to compare with one of the most complete and comprehensive data set on pion production at high energy. Such data were collected by the HARP experiment at CERN [30, 31], where extensive measurements of double-differential cross sections for charged-pion production in proton- and pion-induced reactions were performed. Incident momenta of $3,5,8$ and $12 \mathrm{GeV} / c$ were considered.

\subsection{Integral pion production}

Figures 35 show inclusive pion-production cross sections integrated over the acceptance of the HARP experiment. In addition to the INCL ++ calculation, we show the results of three other models: Bertini [29] and Binary Cascade (BIC) 28, are popular intranuclear-cascade models available in Geant4, while INCL4.6 represents the Liège Intranuclear Cascade model without multipion extension 32. The difference between INCL4. 6 and INCL ++ clearly highlights the importance of the extension, which is already sizable at the lowest incident momentum of the HARP data-set $(3 \mathrm{GeV} / c)$. The INCL4.6 model is reported to illustrate certain surprising features of the hadronic shower in Sec. 4, namely the relative insensitiveness to the details of the treatment of the individual elementary interactions.

The INCL ++ and Bertini models provide comparably accurate predictions. Bertini is generally closer to the experimental data for light targets, while INCL ++ performs better on heavy targets, such as lead, which is most interesting for the present work and in general for spallation neutron sources. Proton-nucleus data are generally better reproduced than pion-nucleus data, with all calculations anyway being within a factor of 2 from the experimental data, with the BIC model having greater difficulties in reproducing the experimental cross sections. 


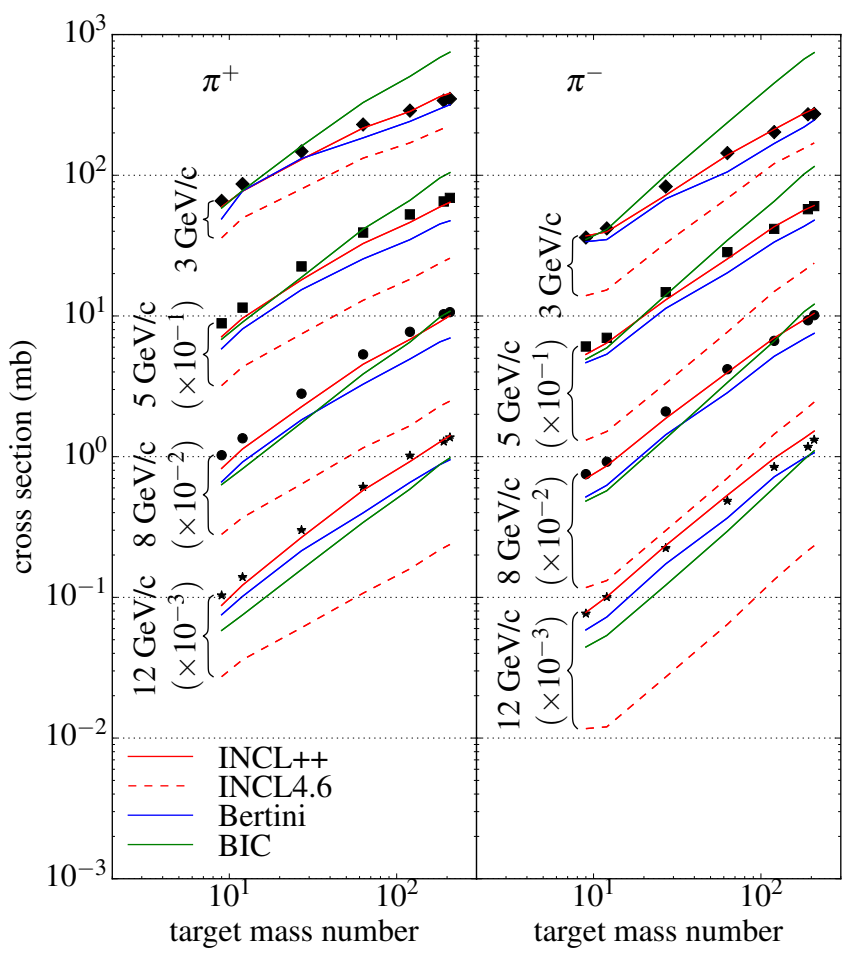

Figure 3. Cross sections for the production of $\pi^{+}$(left) and $\pi^{-}$(right) from proton-nucleus reactions, integrated over the HARP angle-momentum acceptance, for different incident proton momenta, as functions of the target mass number. The lines represent calculations by different models (see text for details). The experimental data are taken from Ref. 30.

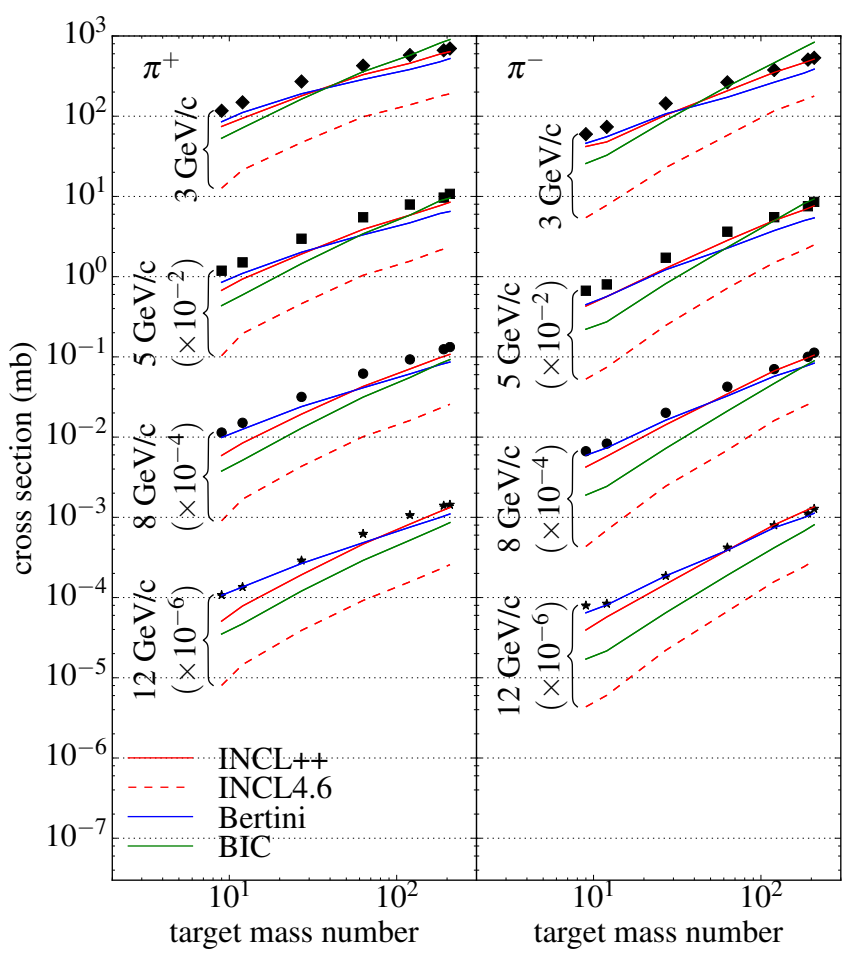

Figure 4. Same as Fig. 3 but for $\pi^{+}$-nucleus reactions.

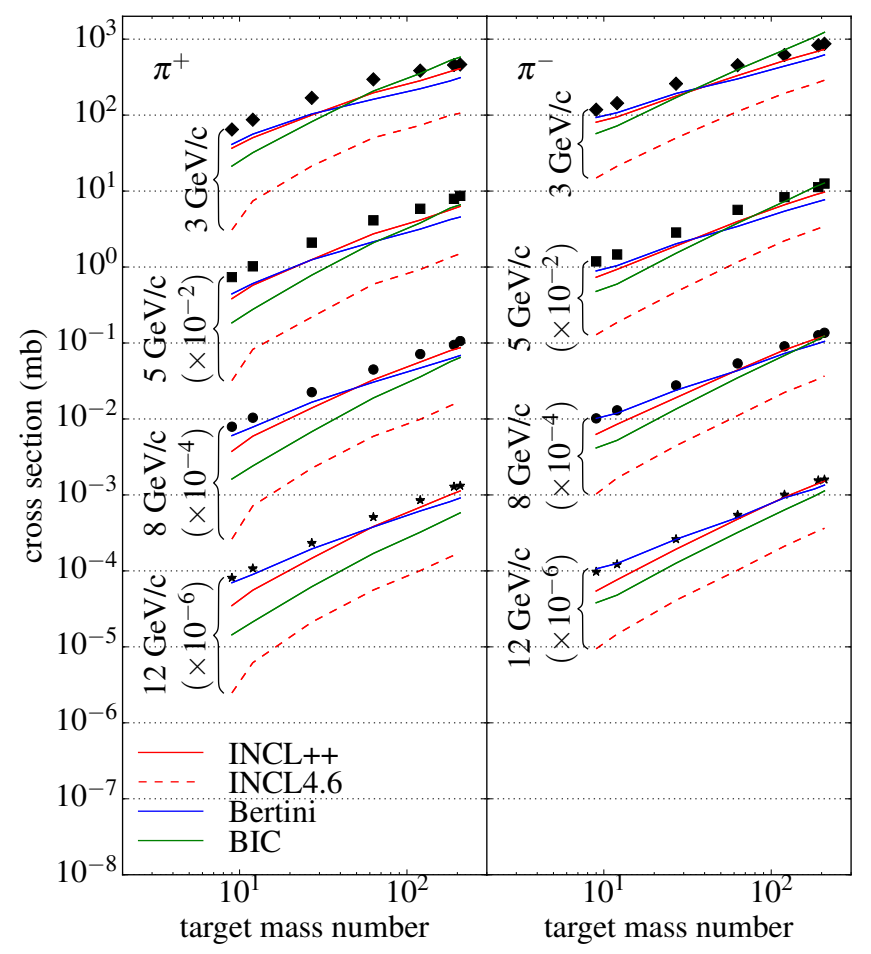

Figure 5. Same as Fig. 3 but for $\pi^{-}$-nucleus reactions.

It is important to remark that very few inclusive experimental data exist for the production of neutral pions in proton-nucleus and pion-nucleus reactions. This is of course mainly due to the short lifetime of the neutral pion, which complicates its detection. It is therefore customary to benchmark reaction models only on charged-pion production. We will follow the same approach in the present work. The validity of the interpolation to neutral pions can often be directly related to the goodness of the isospinsymmetry approximation, which is commonly used for the computation of elementary cross sections in intranuclear cascade.

\subsection{Double-differential pion-production cross sections}

Figures 6 and 7 show double-differential (momentum-angle) cross sections for inclusive pion production in proton- and pion-induced reactions. For benchmarking we select two incident momenta - 3 and $12 \mathrm{GeV} / c$ - and we focus on the lead target, which is the most important for the study of the $n$ TOF spallation source. For simplicity, we limit our discussion to $\pi^{+}$production in proton- and $\pi^{-}$-induced reactions; these results exhibit all the typical features of the general case.

For the purpose of this work, the most interesting quantity to compare is the pion emission spectrum. The cross sections of Figs. 35 are determined by integration of the double-differential cross sections in Figs. 6 and 7 over the momentum and angle acceptance of the HARP data-set. It clearly appears that no model accurately reproduces the emission spectra for all angles and momenta. INCL ++ 


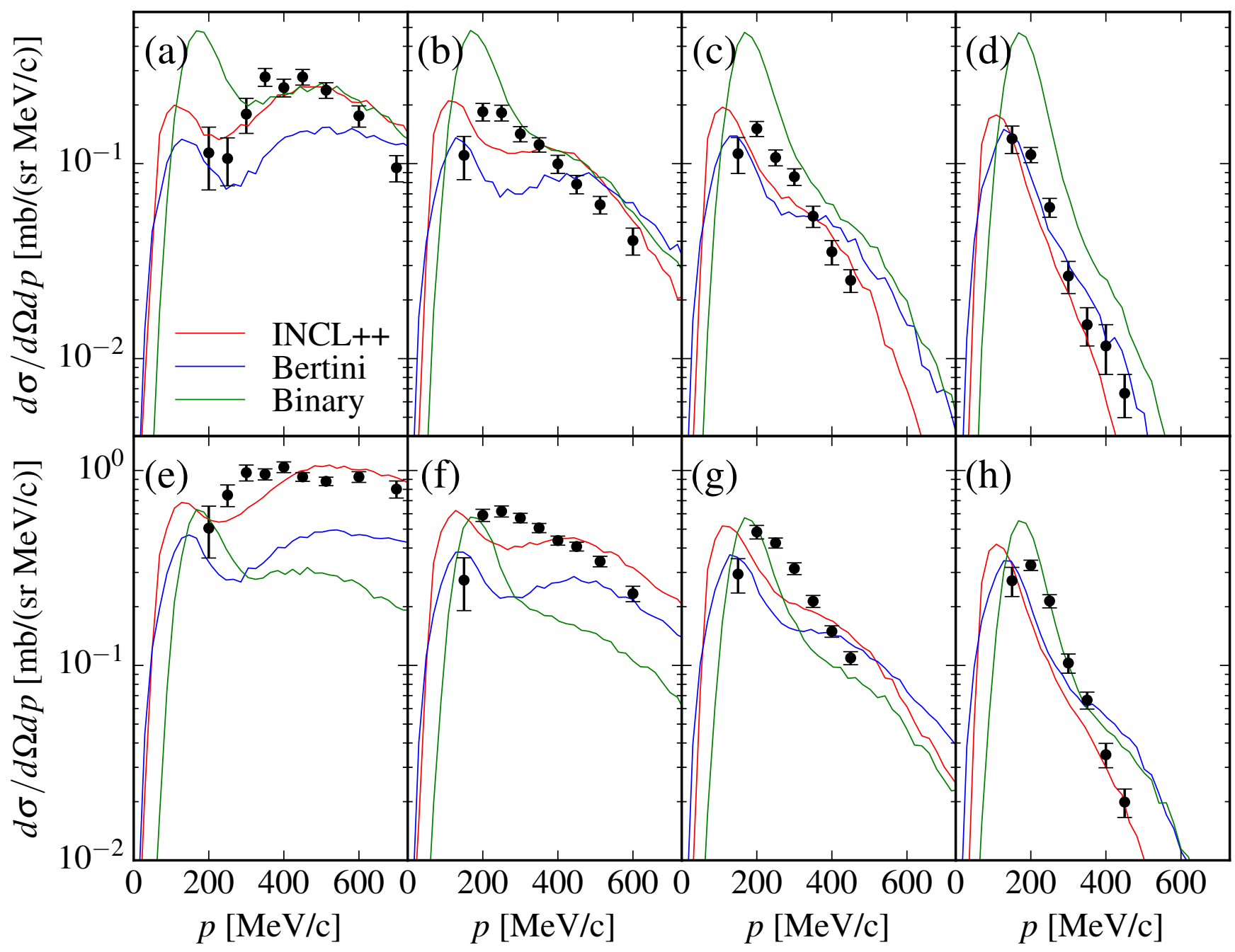

Figure 6. Double-differential cross sections for the production of $\pi^{+}$at $25^{\circ}$ (a, e), $48^{\circ}(\mathrm{b}, \mathrm{f}), 71^{\circ}$ (c, g) and $105^{\circ}(\mathrm{d}, \mathrm{h})$, from $3 \mathrm{GeV} / c(\mathrm{a}-\mathrm{d})$ and $12 \mathrm{GeV} / c(\mathrm{e}-\mathrm{h}) \mathrm{p}+\mathrm{Pb}$. The lines represent calculations by different models (see text for details). The experimental data are taken from Ref. 30.

and Bertini are generally more accurate at forward and backward angles, respectively, while BIC is, as already noted, rather far from the experimental data. The goodness of the model predictions for this observable is qualitatively consistent with the results for neutron production in Geant4 simulations of the $\mathrm{n}$ - TOF spallation target, providing further evidence of the fundamental role of pion-induced reactions in thick spallation targets.

An interesting observation that can be made about Figs. 6 and 7 is that INCL ++ and Bertini consistently show a dip in the spectra at forward angles (even up to roughly $90^{\circ}$ ) and around $250 \mathrm{MeV} / \mathrm{c}$, which is not seen in the experimental data. This defect was also noticed by the authors of Ref. [29, who tentatively attributed it to insufficient moderation by the nuclear medium. In our opinion, however, the dip is related to the formation and decay of the $\Delta(1232)$ resonance, which manifests itself as a strong peak in the pion-nucleon cross section. This intuition is triggered by the observation that the position of the dip coincides approximately with the position of the resonance in the $\pi+N \rightarrow \Delta$ cross section. Indeed, we have verified that the dip is insensitive to reasonable modifications of the recombination $(\Delta+N \rightarrow N+N)$ cross section.

The mechanism leading to the formation of the dip in the model is rather simple, if one makes a few reasonable assumptions. First, we assume that pion production in INC proceeds in two stages. In the first stage, early elementary collisions generate a structureless (no dip) pion spectrum (it is reasonable to assume that pions are produced early in the reaction because the energy available for pion production quickly degrades after a few collisions). In the second stage, the generated pions traverse the nucleus, possibly undergoing scattering and absorption, and possibly emerging as free particles. In this picture, the early pions are attenuated by the nuclear medium, with the excitation of the $\Delta(1232)$ resonance playing a role in the distortion of the pristine pion spectrum, due to selective pion absorption at the corresponding resonance energy. Since 


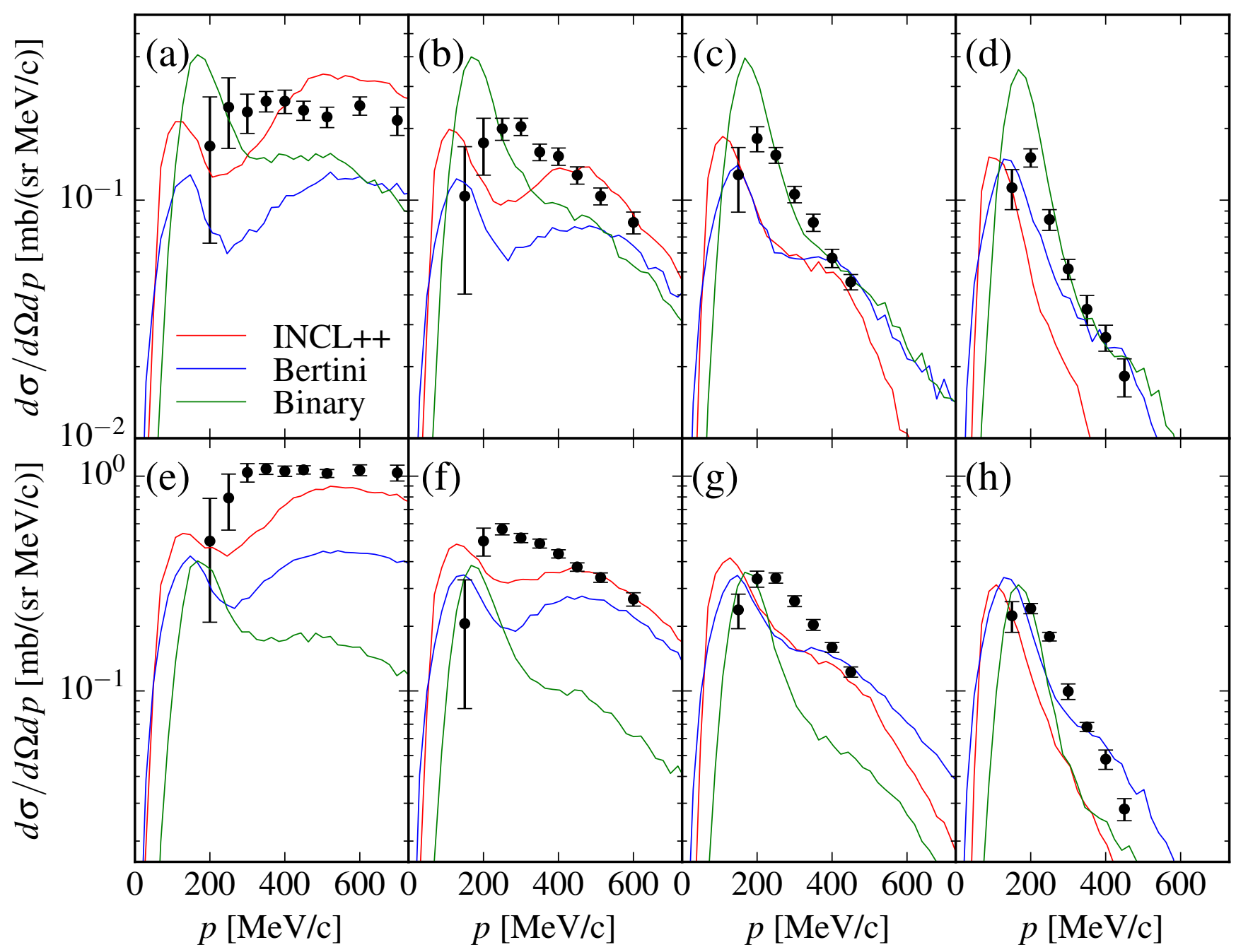

Figure 7. Same as Fig. 6. but for $3 \mathrm{GeV} / c(\mathrm{a}-\mathrm{d})$ and $12 \mathrm{GeV} / c(\mathrm{e}-\mathrm{h}) \pi^{-}+\mathrm{Pb}$ reactions.

the dip is insensitive to the recombination cross section and to the resonance lifetime, and since $\Delta$ resonances (in INCL) can only be absorbed by recombination, we conclude that the intermediate $\Delta$ resonances mostly decay back to pion-nucleon pairs. In principle, the momentum of the pion should fall back in the dip region. However, while the formation and decay of the intermediate $\Delta$ resonances does not modify the pion momentum distribution, it does act on the angular distribution. If one makes the reasonable assumption that the pristine pion spectrum is sensibly forward-peaked, then the decay of the intermediate $\Delta$ resonances will redistribute pions from the forward angles to all angles. This manifests itself as a dip at forward angles in the double-differential spectra.

While this explanation might hold valid for the dip observed in the model calculations, it is not clear whether it also applies to the data. A hint of a dip may be seen in the very forward angles, but in general data seems to indicate that in reality the dip, if any, is less pronounced than what predicted by the models. We performed some tests and we verified that the dip disappears if the $\pi+N \rightarrow \Delta$ cross section is artificially reduced by about a factor of 2 . One can also act on the width of the $\Delta$ resonance peak in the $\pi+N \rightarrow \Delta$ entrance channel: interestingly, either increasing or decreasing the width of the Breit-Wigner-like peak will suppress the dip in the calculations. Theoretical calculations [e.g. 33] indicate that in-medium $\Delta$ resonances should be broader than the corresponding free particles, although unambiguous quantitative indications are still missing 34. INCL already generates part of this medium effect (on the resonance lifetime) through the application of Pauli blocking on the resonance decay and through $\Delta$ absorption. For consistency one should also modify the cross section of the formation process to reflect this. It remains to be seen if a realistic modification of the $\Delta$ width (in the spirit of e.g. Refs. 35, 36) can reconcile the calculations with the experimental data.

For the sake of completeness, we mention that there is disagreement about the scientific adequateness of the HARP data analysis. A group of former HARP collaborators (the HARP-CDP group) have published a revisited analysis of the raw HARP data, which has sparked a long 


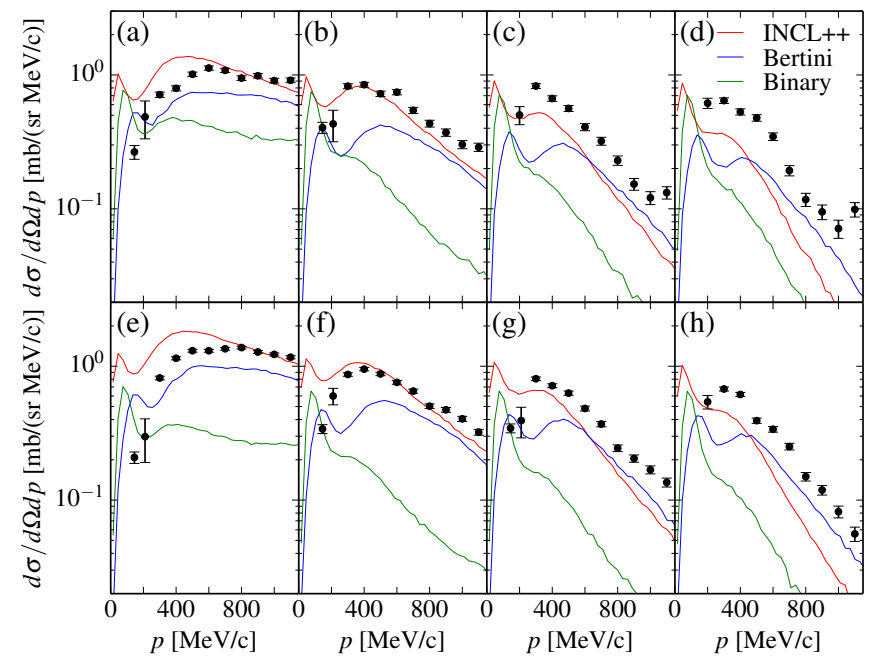

Figure 8. Double-differential cross sections for the production of $\pi^{+}$at $0^{\circ}-25.8^{\circ}(\mathrm{a}, \mathrm{e}), 25.8^{\circ}-41.0^{\circ}(\mathrm{b}, \mathrm{f}), 41.0^{\circ}-50.6^{\circ}(\mathrm{c}, \mathrm{g})$ and $50.6^{\circ}-59.0^{\circ}(\mathrm{d}, \mathrm{h})$, from $12.3 \mathrm{GeV} / c(\mathrm{a}-\mathrm{d})$ and $17.5 \mathrm{GeV} / c$ $(\mathrm{e}-\mathrm{h}) \mathrm{p}+\mathrm{Au}$. The lines represent calculations by different models (see text for details). The experimental data are taken from Ref. 39

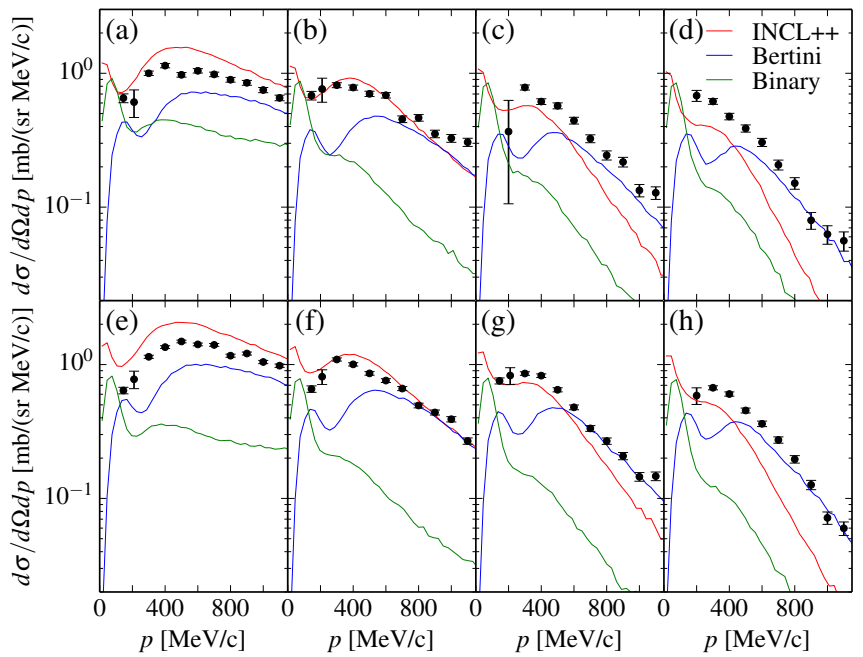

Figure 9. Same as Fig. 8, but for the production of $\pi^{-}$.

and well-documented controversy 37. Ref. 38 contains direct comparisons of the double-differential momentumangle cross sections, but only for the smallest angle $\left(25^{\circ}\right)$ and for 3 and $8 \mathrm{GeV} / c$ beam momenta (Figs. 12 and 13 in their paper). On this limited basis, it is difficult to decide whether the HARP-CDP cross sections are compatible with the dip in the calculations, although the fact that the HARP-CDP data seem to be consistently smaller than the HARP data at low momentum is encouraging.

Given this state of affairs, it is surely wise and instructive to consider other data-sets. Figures 8 and 9 show results for the calculation of double-differential pion-emission cross sections for 12.3 and $17.5 \mathrm{GeV} / c$ protons on gold targets, compared to the data from Ref. 39. The energy and the target for the $12.3 \mathrm{GeV} / c$ data-set are close to

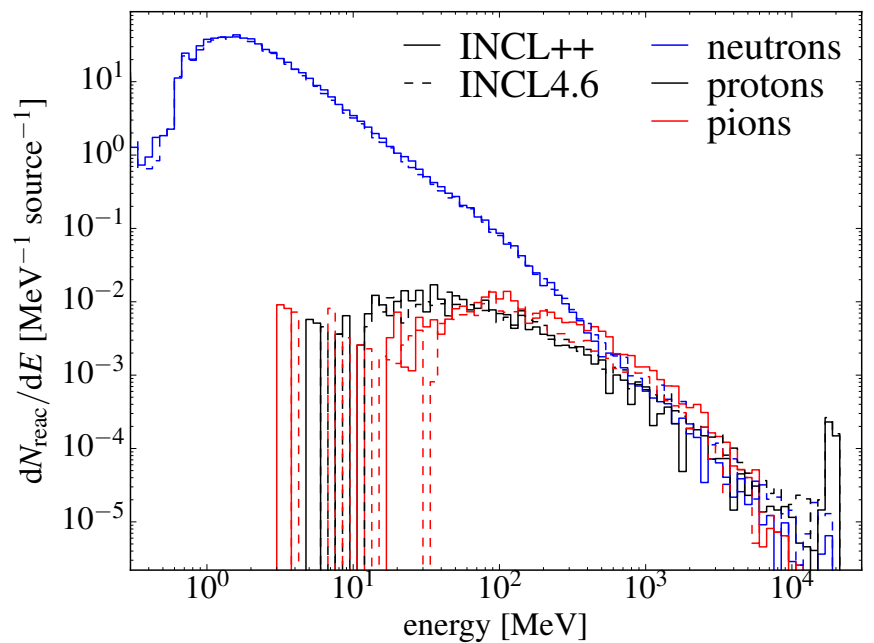

Figure 10. Incident-energy distribution of nuclear reactions induced within the hadronic cascade by protons, neutrons and pions, normalized to one primary proton, as calculated by the INCL model with (INCL ++ , solid lines) and without (INCL4.6, dashed lines) multipion extension, within the Geant4 simulation of the $\mathrm{n}$ - TOF spallation target (beam momentum of $20 \mathrm{GeV} / c$ ).

the HARP data, Figs. 6 and 7. However, when comparing the two data-sets, it is important to keep in mind that 1) the momentum acceptance is larger in Chemakin's data, and 2) the measured angles are smaller: the largest measurement angle in Chemakin's data-set falls between the second and the third HARP measurement angle. If one makes abstraction of these differences, the models appear to behave consistently over all the Figs. 6 9. Therefore, we do not see any clear indication that the HARP data should be rejected.

In view of these difficulties, new, high-acceptance data focusing on the pion production in high-energy protoninduced reaction would be highly desirable. Together with charged pions, direct measurements of $\pi^{0}$ production would provide fundamental information that could contribute considerably to the optimization of the INC models.

\section{Thick target: pions in the $n$ TOF spallation target}

While thin-target double-differential cross section can provide some indications on the ability of the models to correctly predict pion production, the structure of the hadronic showers that take place in the spallation target, and in particular the role played by secondary pions in the production of neutrons and photons, can only be studied by means of dedicated MC simulations of the spallation process and comparison with available experimental data. In this respect, we have chosen in this work to perform further simulations of the $n_{-}$TOF spallation target with the Geant4 toolkit. Before analysing the results of the simulations, it is convenient to briefly describe the toolkit and 
the implementation of the MC simulations for the $\mathrm{n}_{-} \mathrm{TOF}$ case.

\subsection{The Geant4 toolkit}

Geant4 (GEometry ANd Transport) is a toolkit for the simulation of particle transport and detector response 12 13. The Geant family of codes was originally developed for the needs of the high-energy-physics community. However, since the beginning the array of physics models has been constantly expanding to encompass applications at lower energy. In particular, Geant4 has been successfully used, since several years, to describe the transport of neutrons down to thermal energy, using point-wise cross-section from evaluated libraries 40 . These developments recently triggered new work on the use of Geant4 for the simulation of spallation neutron sources. Recently, Geant4 simulations performed for the $\mathrm{n}$ TOF source were benchmarked against experimental results [10, such as the neutron fluence and resolution function, and yielded interesting results which will be shortly described in the following subsection.

Physics models in Geant4 are organized and collected in "physics lists", which are specifications of the physical processes (and the associated models) that should be used in the simulation. The names of the available Geant4 physics lists are often obtained by concatenating the names of the models used in the hadronic sector, in decreasing order of incident energy. Thus, for instance, the FTFP_INCLXX_HP physics list, around which much of the present work revolves, relies on the Fritiof + preequilibrium model (FTFP) at high energy, the INCL ++ model at intermediate energies, and the NeutronHP model at low energy.

This work is based on Geant 4 v10.1; however, the INCLmodel within Geant4 was manually upgraded to v5.2.9.4, which is the version that has been distributed with Geant4 v10.2 (December 2015).

\subsection{The $n$ _TOF simulation}

The $n_{-}$TOF spallation target is a water-cooled lead cylinder surrounded by an aluminum container and by a neutron moderator. Its structure is described in detail in Refs. 8 , 10, 11. A $20 \mathrm{GeV} / c$ proton beam impinges on the base of the lead cylinder at an angle of approximately $10^{\circ}$. The lead target cylinder can be considered as thick, in the sense that its size (radius $30 \mathrm{~cm}$, length $40 \mathrm{~cm}$ ) is large compared to the proton mean free path for inelastic collisions at the beam energy $(\sim 15 \mathrm{~cm})$. Therefore, the primary proton often triggers a nuclear reaction inside the $\mathrm{Pb}$ target and initiates a hadronic shower which eventually leads to the production of a large number of particles. A note about our nomenclature: we refer to all "non-primary" particles as secondaries, regardless of the reaction generation they appear in, in opposition to the "primary" particle incident on the spallation target.
Among the particles escaping from the spallation target, we are particularly interested in neutrons, which are moderated in water, that can be either normal or borated, and collimated towards the experimental areas. Simulations of the $n_{-}$TOF spallation target have focused on the reproduction of the measured energy dependence, resolution function, and spatial distribution of the neutrons entering the first experimental area (EAR1) [8, 10. The expected flux in the direction of the second, new experimental area (EAR2) was also studied in a recent paper [11. As shown in Refs. 10, 11, these measured quantities are best reproduced by the simulations using the FTFP INCLXX HP and QGSP INCLXX HP physics lists. For proton-nucleus reactions, these physics lists use the Liège Intranuclear Cascade model $(\mathrm{INCL}++)$ from $1 \mathrm{MeV}$ to $20 \mathrm{GeV}$ incident energy, and either the Fritiof + pre-equilibrium model (FTFP) or the Quark-GluonString + pre-equilibrium model from $15 \mathrm{GeV}$ upwards. In the region where the two model overlap $(15-20 \mathrm{GeV})$, the choice of the model is randomly sampled, with linearlyinterpolated probabilities between the interval endpoints (a standard procedure in Geant4). Since the primary proton beam energy is $\sim 19 \mathrm{GeV}(20 \mathrm{GeV} / c)$, it is clear that the FTFP or QGSP will typically be used at most for the simulation of the first inelastic proton-nucleus reaction 3 the rest of the hadronic shower is dominated by the INCL ++ model. For this reason, we limit our analysis on the FTFP INCLXX HP physics list. The following section introduces a global description of INCL ++ , with particular focus on its pion dynamics.

\subsection{Analysis of secondary reactions}

We start by illustrating how nuclear reactions in the target are distributed with respect to the type of the incident particle and its energy. Figure 10 shows the distribution of the projectile energy for nuclear reactions induced by protons, neutrons and pions. Each distribution is normalized to the total number of reactions per primary proton induced by the indicated particle. Note that Fig. 10 includes the reactions induced by the primary protons, which appear as a small peak close to the beam energy, whose integral roughly amounts to 0.91 reactions per incident proton; this is consistent with the thickness of the spallation target, which is of the order of 2.5 nuclear mean free paths at the beam energy. Note also that this plot does not include elastic collisions. This choice mainly follows from the consideration that elastic scattering on lead nuclei does not sensibly modify the projectile energy for nucleons and pions; in addition, at high energy, the elastic angular distribution is sensibly forward-peaked and is unlikely to affect the global flow of energy and momentum within the spallation target.

Table 1 presents the integral reaction rates over selected ranges of incident energy, as calculated by Geant4 simulations using the INCL model with $(\mathrm{INCL}++)$ and

\footnotetext{
3 Except of course for events of specific classes, involving small energy losses, like e.g. quasi-elastic scattering.
} 


\begin{tabular}{c|ccc|ccc|ccc}
$\begin{array}{c}\text { energy } \\
(\mathrm{MeV})\end{array}$ & neutrons & $\begin{array}{c}\text { INCL++ } \\
\text { protons }\end{array}$ & pions & neutrons & INCL4.6 & \multicolumn{3}{c}{ difference $(\%)$} \\
protons & pions & neutrons & protons & pions \\
\hline $0-1$ & 14.4 & 0 & 1.88 & 13.3 & 0 & 1.40 & 8.7 & 0 & 33.7 \\
$1-20$ & 157.5 & 0.08 & 0.03 & 144.5 & 0.08 & 0.02 & 9.0 & 4.3 & 33.0 \\
$20-200$ & 28.3 & 1.33 & 1.59 & 25.9 & 1.35 & 1.26 & 9.2 & -1.5 & 25.6 \\
$>200$ & 3.51 & $2.60(1.67)$ & 3.70 & 4.20 & $3.07(2.14)$ & 2.57 & -16.5 & $-15.6(-22.1)$ & 44.1 \\
\hline total & 203.7 & $4.01(3.07)$ & 7.20 & 187.9 & $4.51(3.56)$ & 5.26 & 8.4 & $-11.0(-13.9)$ & 36.8
\end{tabular}

Table 1. Average number of reactions per incident proton induced by the indicated particles within the specified energy ranges, as calculated by our Geant4 simulation with (INCL ++ ) and without (INCL4.6) multipion extension. The last three columns show the relative difference between the INCL ++ and INCL4.6 calculations. The numbers within brackets in the proton columns refer to reactions induced by secondary protons alone.

without (INCL4.6) multipion extension. The most important feature of Table 1 and Fig. 10 is that most of the reactions are actually neutron-induced reactions at relatively low energy. This is essentially due to two facts: first, as Fig. 1 shows, neutrons are by far the particles that are most abundantly produced in spallation reactions (note that the neutron curve has been rescaled by a factor 0.25 ). Second, unlike protons and charged pions, neutrons are not stopped by continuous energy loss, so their flight through the target is always terminated either by leakage or by a collision. An additional remark that can be made about the neutron distribution in Fig. 10 regards the structures around $1 \mathrm{MeV}$, which correspond to the opening of $\left(n, n^{\prime}\right)$ reactions on the four lead isotopes, while reactions below the thresholds are dominated by radiative capture on lead isotopes, on the moderating water, as well as on $(n, \alpha)$ reactions on boron. All these features of neutron transport in thick spallation targets are well known.

It is instead surprising that secondary reactions induced by pions are twice as many as those induced by protons. Table 1 shows a contribution to pion-induced reactions even in the range $0-1 \mathrm{MeV}$; this actually corresponds to the absorption of stopped negative pions, whose fate is to quickly form pionic atoms, decay towards the inner atomic shells and eventually disappear in a reaction with the nucleus. The lifetime for the whole process (i.e. formation of the pionic atom, atomic decay and pion absorption) is of the order of $10^{-10} \mathrm{~s}$ [41, which is much smaller than the pion intrinsic lifetime $\left(2.6 \cdot 10^{-8} \mathrm{~s}\right)$. Hence, the decay of stopped negative pions is actually a rare process. In some sense this actually makes negative pions akin to neutrons, insofar as they are bound to induce a nuclear reaction, or escape from the spallation volume.

Even if one neglects $\pi^{-}$absorption at rest, pion-induced reactions are still more common than proton-induced reactions. This finding clashes with the typical validation strategy applied to spallation-reaction modeling at lower energies [5 7], which emphasizes the role of proton-induced reactions. In particular, while this approach is appropriate at ADS energies $(\sim 500-1000 \mathrm{MeV})$, it is clearly insufficient at higher energies, such as those involved in the $n_{-}-$ TOF spallation source. Of course the choice of the validation endpoints is also conditioned by the wide availability of comprehensive data-sets for proton-induced reactions and the relative scarceness of neutron- and pion-induced data.

There is one further interesting remark that can be made about Fig. 10, and especially Table 1. By comparing the numbers for the two models, one notices that pions induce about $35 \%$ more secondary reactions in INCL ++ than in INCL4.6. This is of course a consequence of the fact that the average pion multiplicity is higher in INCL ++ than in INCL4.6. It is however instructive to contrast this $35 \%$ difference with the much larger difference in average multiplicity (Fig. 1), which can be as large as a factor of 8 or 9 . If we take the number of pion-induced reactions as a measure of the number of pions that participate in the hadronic cascade, this result suggests that the structure of the cascade is relatively insensitive to the average pion multiplicity predicted by the nuclearreaction model for the individual reactions. As we shall see in Sec. 4.7 this has important consequences for the sensitivity of secondary-particle yields (neutrons and photons in particular) to the details of the nuclear-reaction models.

\subsection{The role of neutral pions}

In Ref. 10, Geant4 simulations of neutron production at the n_TOF spallation target, performed with several physics lists, were compared between each other and to the energydifferential neutron fluence measured at $n$ TOF in various experimental campaigns. As previously mentioned, it was shown that the lists based on the INCL ++ model closely reproduce the energy dependence of the neutron spectrum and, within a $15-20 \%$ difference, the integrated yield. All other models are able to reproduce the shape, but overestimate the yield by a larger factor, of up to $70 \%$. A different behavior was instead observed for the production of prompt high-energy photons, i.e. those reaching the experimental area within $1 \mu \mathrm{s}$ of the beam pulse, and with an energy larger than $10 \mathrm{MeV}$. In particular, the most and least intense photon yields are respectively predicted by the INCL ++ and Bertini models. In general, an anticorrelation was observed between neutron and promptphoton production, with models predicting larger neutron yields predicting smaller photon yields. Following this observation, several tests were performed with Geant4 simulations in order to understand the origin of the differences 


\begin{tabular}{|c|c|c|c|c|c|c|c|c|}
\hline & & & $\begin{array}{c}\text { FTFP_-} \\
\text { INCL46_HP }\end{array}$ & $\begin{array}{c}\text { FTFP_- } \\
\text { INCLXX_HP }^{-}\end{array}$ & $\begin{array}{c}\text { QGSP } \\
\text { INCLXX_HP }\end{array}$ & $\begin{array}{l}\text { FTFP } \\
\text { BERT_HP }_{-}\end{array}$ & $\begin{array}{l}\text { QGSP }^{\text {QERT_HP }} \\
\text { BERT }\end{array}$ & $\begin{array}{l}\text { QGSP } \\
\mathrm{BIC}_{-} \mathrm{HP}\end{array}$ \\
\hline (a1) & & number $\pi^{0}$ & 4.2 & 6.3 & 6.7 & 5.4 & 5.5 & 6.1 \\
\hline (a2) & & number $\pi^{ \pm}$ & 7.5 & 11.0 & 11.5 & 10.2 & 10.0 & 11.5 \\
\hline (b1) & & reference & 80.7 & 119.0 & 138.0 & 93.0 & 104.4 & 115.4 \\
\hline (b2) & है & no $\pi^{0}(\%)$ & 6.1 & 4.7 & 7.5 & 6.5 & 9.6 & 11.6 \\
\hline (b3) & +0 & no $\pi^{ \pm}(\%)$ & 98.8 & 83.7 & 83.3 & 94.2 & 95.3 & 84.9 \\
\hline (b4) & है & $\pi^{0} \rightarrow \pi^{ \pm}(\%)$ & 7.5 & 4.7 & 7.7 & 7.0 & 10.5 & 11.7 \\
\hline (c1) & & reference & 454.6 & 513.0 & 420.9 & 658.9 & 575.9 & 531.7 \\
\hline (c2) & อี & no $\pi^{0}(\%)$ & 97.4 & 94.5 & 95.2 & 97.1 & 100.4 & 100.8 \\
\hline (c3) & 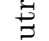 & no $\pi^{ \pm}(\%)$ & 60.1 & 56.2 & 42.9 & 58.1 & 54.1 & 45.3 \\
\hline$(\mathrm{c} 4)$ & $\underset{\Xi}{\Xi}$ & $\pi^{0} \rightarrow \pi^{ \pm}(\%)$ & 125.5 & 128.0 & 138.3 & 112.6 & 133.2 & 132.4 \\
\hline (d1) & & $\pi^{0} \rightarrow \gamma$ & 17.9 & 18.0 & 19.0 & 16.1 & 17.3 & 16.7 \\
\hline$(\mathrm{d} 2)$ &.$\stackrel{0}{0}$ & $\pi^{ \pm} \rightarrow \gamma$ & 0.1 & 1.8 & 2.0 & 0.5 & 0.5 & 1.5 \\
\hline (d3) & $\stackrel{0}{\supset}$ & $\pi^{0} \rightarrow n$ & 2.8 & 4.5 & 3.0 & 3.5 & -0.5 & -0.7 \\
\hline$(\mathrm{d} 4)$ & हี & $\pi^{ \pm} \rightarrow n$ & 24.1 & 20.4 & 21.0 & 27.1 & 26.4 & 25.4 \\
\hline
\end{tabular}

Table 2. Results of test calculations with suppressed $\pi^{0}$ decay, suppressed $\pi^{ \pm}$transport, and $\pi^{0} \rightarrow \pi^{ \pm}$conversion. Columns represent different physics lists (FTFP INCL46 HP being short for FTFP INCLXX HP with INCL4.6). The meaning of the rows is the following: (a1) number of $\pi^{0}$ produced in the reference calculation; (a2) the same, for $\pi^{ \pm}$; (b1-b4) number of produced photons above $10 \mathrm{MeV}$ (b1) in the reference calculation, (b2) without $\pi^{0}$ decay, (b3) without $\pi^{ \pm}$transport, and (b4) with $\pi^{0}$ converted to $\pi^{ \pm} ;(\mathrm{c} 1-\mathrm{c} 4)$ same four lines for produced neutrons; (d1-d4) conversion ratios from $\pi^{0}$ or $\pi^{ \pm}$to photons or neutrons. Rows (a1-a2), (b1), (c1) and (d1-d4) are averages per incident proton; the statistical uncertainties on these values are of the order of a few percent. Rows $(\mathrm{b} 2-\mathrm{b} 4)$ and $(\mathrm{c} 2-\mathrm{c} 4)$ are expressed as percentages of the respective reference result. See text for the exact definition of these quantities. The same results are also reported in graphical form in Fig. 11.

among the available intranuclear cascade models for neutron and prompt-photon production.

In order to shed some light on the mechanisms that leads to the production of photons, we have performed simulations by inhibiting $\pi^{0}$ decay; this allows to establish how often prompt photons originate, either directly or through secondary electromagnetic cascade, from $\pi^{0}$ decay. A few low-statistics test runs were performed with different physics lists. The results are shown in Table 2 which reports the number of produced $\pi^{0} \mathrm{~s}$ (line (a1)), along with the number of photons and neutrons produced in the target, with and without $\pi^{0}$ decay (lines (b1-b2) and $(\mathrm{c} 1-\mathrm{c} 2))$. All numbers are normalized to the number of incident protons. The same results are reported in graphical form in Fig. 11.

Two minor notes of warning. First, all particles emerging from the reaction vertex are counted, regardless of the reaction stage that produced them (intranuclear cascade or statistical de-excitation). Second, the particles are counted when they are produced, i.e. at the reaction site; therefore, in a sense the same particle can contribute more than once if the reaction cascade it initiates spans several collisions within the target. Nevertheless, for the sake of conciseness, we shall make use of expressions such as "the average number of photons originating from $\pi^{0}$ decay"; this is a slight abuse of terminology. However, for the purpose of ascertaining the influence of pions on photon and neutron production, this is inconsequential.

Several considerations can be made on the basis of the results in Table 2 and Fig. 11. First, at least $90 \%$ of the prompt photons descend in some way from $\pi^{0}$ decay. This is clearly indicated by the effect of the suppression of $\pi^{0}$ decay in all the physics lists, but also by the observation that physics lists predicting large $\pi^{0}$ production rates typ- ically also predict larger numbers of photons. The average $\pi^{0}$-to- $\gamma$ conversion factor (line $(\mathrm{d} 1)$ ) amounts to about 17 photons per $\pi^{0}$ decay. Second, the influence of $\pi^{0}$ decay on neutron production (line (c2)) is sensibly smaller and of the order of the statistical uncertainty on the reported neutron counts (a few percent). This is easily understood because the coupling from photon transport to neutron transport is weak; the $\gamma \rightarrow \mathrm{n}$ photonuclear conversion is in general quite inefficient at producing neutrons, compared to pion-induced reactions.

It is then quite clear that photon yields are positively correlated with (and actually dominated by) $\pi^{0}$ production. However, note also that, in the reference calculations, large $\pi^{0}$ production rates (line (a1)) correlate well with small neutron yields (line (c1)), with FTFP_INCLXX_HP (the physics list that best reproduces the $\bar{n}$ _TOF neutron spectrum) predicting in particular the highest $\pi^{0}$ production rate and the lowest neutron yield. This behavior clashes with the intrinsic pion-to-neutron balance of spallation reactions. In the framework of INC models, larger values of the pion multiplicity entail more efficient dissipation of the projectile energy; thus, larger excitation energies are produced at the end of INC, which results in larger evaporation yields. Therefore, pion and neutron multiplicities are in principle positively correlated. The INCL ++ model can be seen to exhibit this behavior in Fig. 1. the multipion extension has indeed the effect of increasing all particle multiplicities. However, while Fig. 1 refers to a thin target case, for a thick target other effects come into play. As discussed later on in this paper, due to the resilience of the hadronic cascade, neutron production in large-volume spallation targets is rather insensitive to the pion multiplicity in individual $N+N$ or $\pi+N$ collisions, but depends mostly on the total number of pions 


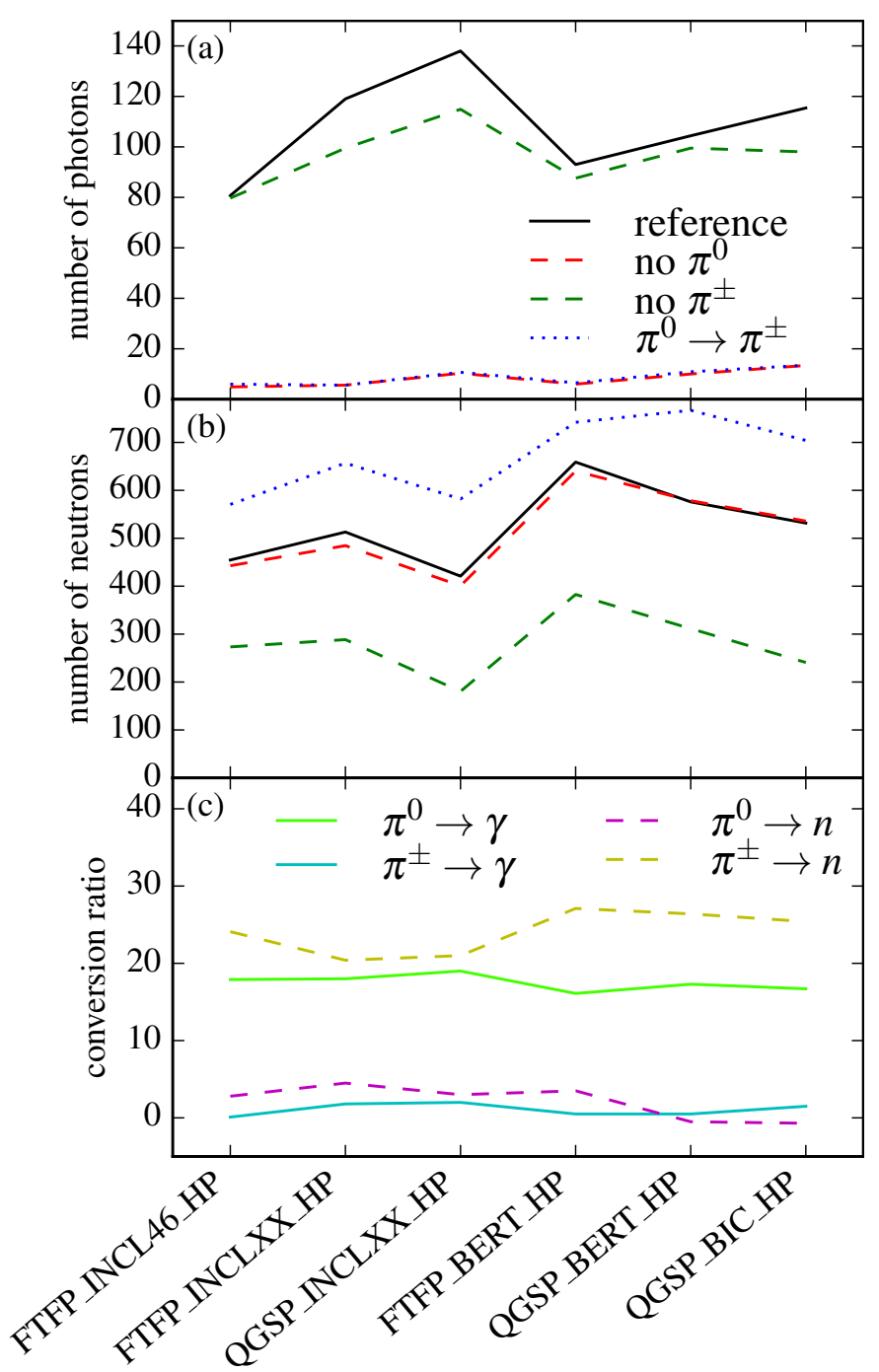

Figure 11. Number of photons (a) and neutrons (b) in the test calculations reported in Table 2 (rows (b1-b4) and (c1c4), respectively), as a function of the physics list used. Panel (c) represents the pion-to-particle conversion ratios (lines (d1d4) of Table 2.

produced in the full hadronic cascade. In particular, for a larger number of pions produced, $40 \%$ of which are $\pi^{0}$, a larger fraction of energy will be diverted from the hadronic cascade to the electromagnetic one, and become unavailable for neutron production. In this sense, the results in Table 2 and Fig. 11 clearly hint to a fundamental role of pion production in general, and $\pi^{0}$ production in particular, in determining the final neutron and photon fluences in spallation neutron sources based on high-energy protons impinging on large spallation volumes.

In summary, it is quite clear that neutral pions dominate the production of prompt, high-energy photons. At the same time, the observed anti-correlation of neutron and prompt-photon yields can be explained considering that neutral pions essentially divert energy from the hadroni cascade towards the electromagnetic one.

\subsection{Influence of charged pions on neutron production}

As we have shown in Fig. 10 and Table 1 above, charged pions are responsible for a large number of secondary reactions. Since spallation reactions often lead to the production of neutrons, one expects that secondary charged pions have a large influence on neutron production.

In order to prove this, we have performed test calculations in which we suppress the transport of charged pions by killing their tracks at the reaction site. This prevents them from inducing further secondary reactions. Table 2 and Fig. 11 show the number of produced $\pi^{ \pm}$tracks (line (a2)), along with the average photon (line (b3)) and neutron yields (line $(\mathrm{c} 3)$ ) recorded when charged pions are not transported. Pion-induced reactions are seen to account for about $40-45 \%$ of the neutron yield. Depending on the physics list, the suppression of one charged pion entails a reduction of 20-25 neutrons per incident proton (line (d4)), with INCL-based and Bertini-based physics lists respectively yielding the lowest (20.4) and highest (27.1) pion-to-neutron conversion ratios. There is also an effect on the photon yields (line (b3)), which also decrease by $5-20 \%$ when charged pions are suppressed. This is probably due to the disappearance of the neutral pions that would have been produced in the suppressed $\pi^{ \pm}$-induced reactions.

To further substantiate our claims about the importance of secondary pions, we have performed test calculations where $\pi^{0}$ production is randomly replaced with the production of a $\pi^{+}$or a $\pi^{-}$(with equal probability) with the same kinetic energy and momentum direction as the suppressed $\pi^{0}$. In this test, energy is essentially conserved (up to the small $\pi^{0} / \pi^{ \pm}$mass difference), and charge is conserved on average. The effect on photon yields (line (b4)) is essentially the same as in the calculation without $\pi^{0}$ decay, which is reasonable; neutron yields (line (c4)), on the other hand, are increased by about $10-30 \%$, depending on the physics lists. Note that the increased neutron yields are consistent with the conversion efficiency that can be estimated from the $\pi^{0} \rightarrow n$ and $\pi^{ \pm} \rightarrow n$ conversion coefficients (lines $(\mathrm{d} 3-\mathrm{d} 4))$ and the number of transformed $\pi^{0}$ (line (a1)).

\subsection{Influence of the physics list}

It is worth spending a few words about the dependence of the numbers in Table 2 on the choice of the physics list. In particular, it is instructive to compare results obtained with the same INC/de-excitation component, but different high-energy reaction models. When doing so, care must be exercised because the switching energy between parton-string models and INC depends on the physics list. For instance, in the case of neutron-nucleus reactions, the INC stage is used up to $9.9 \mathrm{GeV}$ in QGSP_BERT_HP, up to $5 \mathrm{GeV}$ in FTFP BERT $\mathrm{HP}$ and up to $20 \mathrm{GeV}$ in QGSP_INCLXX_HP and FTFP_INCLXX_HP.

Therefore, we focus on the most straightforward comparison, namely the one between QGSP_INCLXX_HP and FTFP_INCLXX_HP, which use the same fade-out 
energy interval between INCL ++ and the parton-string model $(15-20 \mathrm{GeV})$. Table 2 shows that QGSP_INCLXX_HP produces $16 \%$ larger photon yields and $\overline{18} \%$ smaller neutron yields. This is qualitatively consistent with the observation by Lerendegui et al. [1] that QGSP_INCLXX_HP yields smaller neutron fluences; however, the numbers in Table 2 are not directly comparable with Lerendegui et al.'s integrated neutron fluences, because our results include counts of unobserved in-target tracks.

The comparison between QGSP_BERT_HP and FTFP BERT_HP leads to the same kind of conclusions, albeit quantitative comparisons are difficult. Inspection of the differences between FTFP_INCLXX_HP and FTFP _BERT HP, for instance, suggests that the influence of the INC/de-excitation stage is of comparable magnitude.

\subsection{Influence of pion multiplicity}

The tests involving the suppression of pion emission might be considered as unrealistic because they grossly violate several conservation laws. Indeed, each pion carries several hundreds $\mathrm{MeV}$ of mass and kinetic energy, which can be (partially) converted into neutrons (through nuclear reactions) or photons (through nuclear reactions or $\pi^{0}$ decay) if the pion is not suppressed. Charge conservation is also violated when charged pions are killed. The $\pi^{0} \rightarrow \pi^{ \pm}$transformation test, on the other hand, approximately conserves energy (up to the $\pi^{0} / \pi^{ \pm}$mass difference), respects charge conservation on average, and provides insight to the sensitivity of the simulation to the pion charge distribution.

It is also instructive to study the dependence of the calculation results on the mean pion multiplicity in individual nuclear reactions. One way to test the influence of multiplicity without abandoning energy conservation is to compare the results of calculations performed with nuclear-reaction models that yield different average pion multiplicities. It is however difficult to isolate the effect of the change in pion multiplicity from all the other differences between the models. Ideally, one would like to change the average pion multiplicity and nothing else. The closest approach to an ideal setting for a sensitivity study is to modify the nuclear-reaction physics within the same model. By acting on some internal model parameter, one could modify the average pion multiplicity while keeping the overall coherence of the model. For this purpose, we shall discuss calculations performed with INCL ++ with and without multipion extension (see Sec. 2). We remind the reader that pion multiplicities are much smaller in the model without multipion extension, starting from about $1 \mathrm{GeV}$ incident energy (Fig. 1).

Figure 12 shows the calculated neutron fluence per unit lethargy and per incident proton emitted from the spallation source in the direction of the first experimental area. This spectrum is scored at the exit of our geometry and we only consider neutrons within a $2^{\circ}$ cone around the direction towards EAR1. This spectrum actually represents the input for the resampling procedure that leads to the

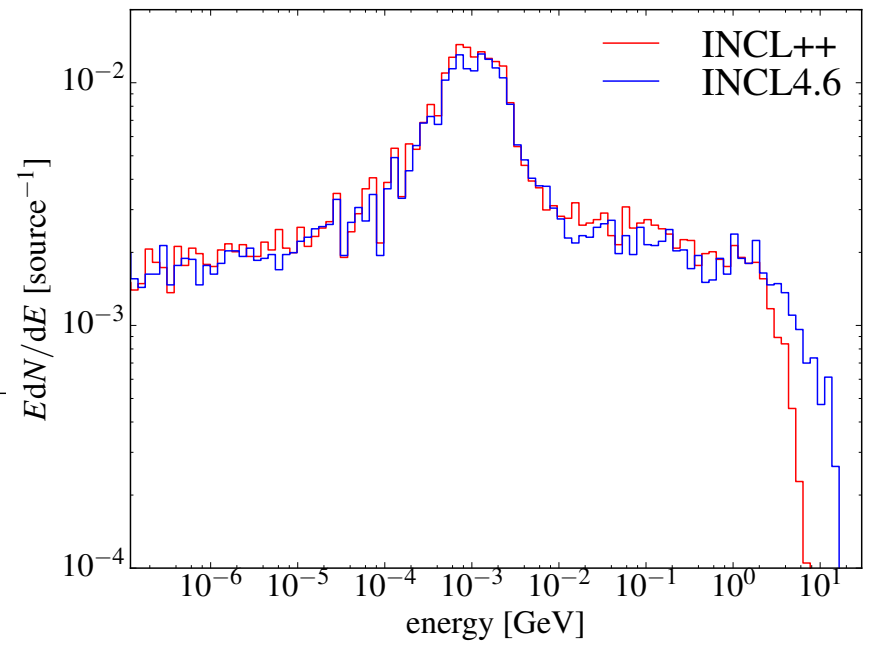

Figure 12. Neutron spectrum per unit lethargy emitted from the $n \_$TOF spallation source in a $2^{\circ}$ cone directed towards the first experimental area, as calculated by our Geant 4 simulation. Results are shown for the INCL model with $(\mathrm{INCL}++)$ and without (INCL4.6) multipion extension.

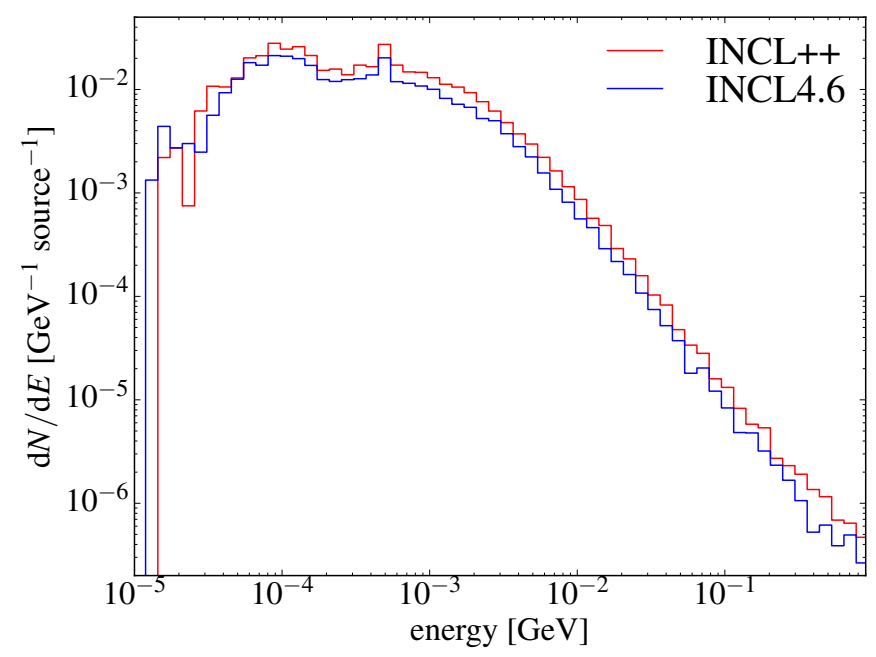

Figure 13. Photon spectrum emitted from the spallation source within $100 \mathrm{~ns}$ of the beam pulse, in a $2^{\circ}$ cone directed towards the first experimental area, as calculated by our Geant4 simulation. Results are shown for the INCL model with $(\mathrm{INCL}++)$ and without (INCL4.6) multipion extension.

\begin{tabular}{c|c|c|c}
$\begin{array}{c}\text { energy } \\
(\mathrm{GeV})\end{array}$ & $\begin{array}{c}\text { INCL }++ \\
\left(\times 10^{-3}\right)\end{array}$ & $\begin{array}{c}\text { INCL4.6 } \\
\left(\times 10^{-3}\right)\end{array}$ & $\begin{array}{c}\text { difference } \\
(\%)\end{array}$ \\
\hline $0-1$ & 72.1 & 66.9 & +7.7 \\
$1-20$ & 2.4 & 3.5 & -31.4 \\
\hline total & 74.5 & 70.4 & +5.8
\end{tabular}

Table 3. Average number of neutrons per incident proton emitted in a $2^{\circ}$ cone directed towards the first experimental area, in the indicated energy ranges, as calculated by our Geant4 simulation. Results are shown for the INCL model with $(\mathrm{INCL}++)$ and without (INCL4.6) multipion extension. 


\begin{tabular}{c|c|c|c}
$\begin{array}{c}\text { energy } \\
(\mathrm{MeV})\end{array}$ & $\begin{array}{c}\text { INCL }++ \\
\left(\times 10^{-3}\right)\end{array}$ & $\begin{array}{c}\text { INCL4.6 } \\
\left(\times 10^{-3}\right)\end{array}$ & $\begin{array}{c}\text { difference } \\
(\%)\end{array}$ \\
\hline $0-10$ & 50.9 & 37.8 & +34.7 \\
$>10$ & 11.6 & 8.0 & +45.0 \\
\hline total & 62.5 & 45.8 & +36.5
\end{tabular}

Table 4. Same as Table 3 , for the emission of photons within $100 \mathrm{~ns}$ of the beam pulse.

estimation of the fluence at EAR1, which is $185 \mathrm{~m}$ away [10].

It is immediately obvious that the difference between the two models is much smaller than the differences seen in the previous section. Table 3 shows the average numbers of neutrons produced in selected energy ranges. The global effect of the multipion extension on the neutron yield is of the order of a few percent. This should be compared to the reduction of $\sim 40 \%$ that was induced by the suppression of the charged-pion tracks.

The neutron spectra are essentially identical in shape up to about $\sim 1 \mathrm{GeV}$. As expected, the shape of the low-energy $(E \lesssim 20 \mathrm{MeV})$ end of the spectrum, which is treated using evaluated cross-section databases, is independent of the high-energy model chain that feeds it. This remark has already been made in connection with the comparison of the spectra predicted by different physics lists [10]. In the case of the comparison between INCL4.6 and INCL ++ , the similarity stretches up to $1 \mathrm{GeV}$ because the two models indeed yield very similar predictions up to this energy (cfr. Fig. 1). Neutrons above $1 \mathrm{GeV}$ are seen to be more abundantly produced by INCL4.6. This is coherent with the aforementioned observation (Sec. 4.4 that higher pion multiplicities lead to more efficient dissipation of the projectile energy; clearly, it is easier to produce high-energy neutrons if less energy is channeled into pion production. In this respect, the validity of the multipion extension could be verified by examining the neutron fluence produced at the $n$ TOF spallation source in the energy region above $1 \mathrm{GeV}$. However, as of now there is no experimental information available, and in any case the measurement of the neutron fluence at such high energy is not straightforward.

Figure 13 and Table 4 compare the results of INCL4.6and INCL ++-based calculations on prompt-photon production. We apply the same $2^{\circ}$ angular cut on the angle of the emitted photon; in addition, we only select photons that are emitted within 100 ns from the beam pulse. This roughly corresponds to the prompt photons that are detected before $1 \mathrm{\mu s}$ at the EAR1 experimental area [10].

The difference between the calculations is much larger than for neutrons, especially for the production of highenergy photons. This is not surprising, given the crucial role that neutral pions are seen to play in the production of prompt high-energy photons. However, compared to the results of Sec. 4.4 above, the magnitude of the effect is somewhat mitigated.

The results shown above indicate that the pion multiplicity in a single nucleon-nucleon or pion-nucleon collision does not play a crucial role in neutron and photon pro-

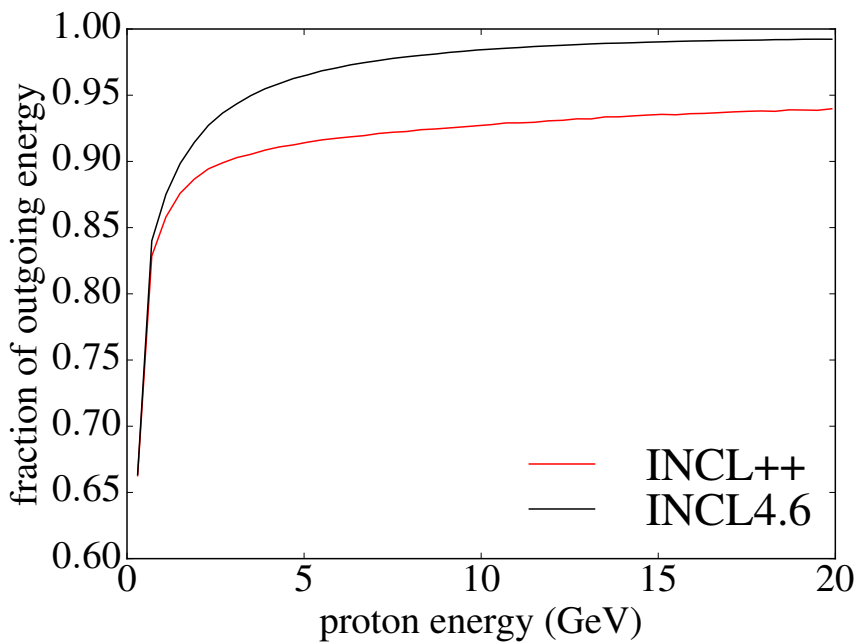

Figure 14. Excitation function for the average fraction of outgoing energy in $\mathrm{p}+{ }^{208} \mathrm{~Pb}$ reactions, as calculated with $(\mathrm{INCL}++)$ and without (INCL4.6) multipion extension. The masses of escaping $\pi^{ \pm}$are counted as outgoing energy. Neutral pions and photons are not counted.

duction in thick spallation targets, contrary to the large effect predicted in the thin target case and shown in Fig. 1. This property has already been observed at lower energies 21] ; our work confirms that it still holds at n_TOF energies. The fact that photon and neutron production are not affected by the reduced pion multiplicity of INCL4.6 (Sec. 4.7) can be partly ascribed to the fact that in this model pions are still produced, albeit not as abundantly as in INCL ++ . However, a more general argument to explain the relative insensitivity of the photon and neutron yields to the details of the INC model involves a rather peculiar feature of thick spallation targets, which can actually be inferred from most thick spallation-target benchmarks [see e.g. 42. In thick spallation targets, in fact, it is the structure of the hadronic cascade that plays a compensatory role.

To better describe the effect, it is also useful to look at the outgoing energy of a reaction (in this case $\mathrm{p}+{ }^{208} \mathrm{~Pb}$ ), defined as the sum of all the kinetic energies of all the reaction products, except photons and $\pi^{0} \mathrm{~s}$. For outgoing $\pi^{ \pm}$, their mass is also counted as outgoing energy. The outgoing energy represents the maximum energy which is liable to be injected in further nuclear reactions (neglecting photonuclear reactions). Figure 14 shows the ratio between the outgoing energy and the incident projectile energy, as a function of the projectile energy, in $\mathrm{p}+{ }^{208} \mathrm{~Pb}$, as calculated with INCL4.6 and INCL ++ . The two models yield very similar predictions, even at $20 \mathrm{GeV}$, with the difference never exceeding $5 \%$ of the incident energy. This similarity should be contrasted with Fig. 1. which demonstrates that the events generated by the two models are radically different.

Of course, the insensitivity of the outgoing energy to the details of the model is partly a trivial consequence of the fact that energy and momentum are conserved in both cases. Yet, the curves in Figure 14 have important conse- 
quences for the development of the hadronic cascade in a thick target. For the sake of clarity we assume here that secondary particles have a mean free path for inelastic collisions which is short with respect to the size of the geometry and, in the case of charged particles, to the range. If this assumption holds, as it may often be the case for thick spallation targets, particles leaving the first reaction vertex are likely to induce secondary reactions nearby, leading to further degradation of the projectile energy. Therefore, for a less dissipative model (such as INCL4.6) it will take a greater number of soft reactions to degrade the projectile energy into low-energy particles. On the contrary, a more dissipative model (such as INCL ++ ) will require fewer hard reactions. However, on a geometric scale larger than the reaction mean free path, the overall structure of the resulting hadronic cascade will be relatively independent of the details of the model. In other words, the number of neutrons and photons produced in the full hadronic cascade (and therefore the fluence of particles emerging from the spallation target) will mostly depend on the total number of pions (and other intermediate particles) produced in the cascade, rather than on their multiplicity in each reaction.

It remains to be seen if the conditions for a short mean free path are met in the case of the $n$ _TOF spallation target. The reaction cross sections for high-energy protons, neutrons and pions in lead are weakly dependent on energy and all of the order of $1.8 \mathrm{~b}$, which results in mean free paths of the order of $15 \mathrm{~cm}$. This is sensibly smaller than the thickness of the spallation target $(40 \mathrm{~cm})$. A 15$\mathrm{cm}$ range in lead corresponds to a proton energy of about $400 \mathrm{MeV}$, or a pion energy of $250 \mathrm{MeV}$. Therefore, for particle energies above roughly $600 \mathrm{MeV}$ (and for neutrons of all energies), the aforementioned conditions are approximately met and the target may be considered as thick.

\section{Conclusions}

Triggered from recent works on Geant4 simulations of the $\mathrm{n}$. TOF spallation target, which indicated some sizable differences in neutron production between various INC models, we have here investigated the role of secondary pions in high-energy proton-induced reactions, both for thin and thick targets. A comparison with the available experimental data points to a large underestimation of pion production by the previous version of the Liège intranuclearcascade model. Some shortcoming is also observed for the Binary and Bertini code in reproducing measured doubledifferential cross sections for charged pion production in $\mathrm{p}+\mathrm{Pb}$ reactions at high energy $(12 \mathrm{GeV} / c)$. On the contrary, the recent version of the Liège Intranuclear Cascade Model, INCL ++ , with multipion extension, reproduce measured cross sections reasonably well at all energies, except for the presence of a dip in the momentum distribution at forward angles (predicted also by the Bertini code). The presence of this dip seems to be related to formation and decay of the $\Delta(1232)$ resonance. However, the scarce data available do not allow at present to draw a conclusion on this effect. Fresh new data in this respect would be highly desirable, together with measurements of neutral pion production, which are essentially missing up to date.

The role of pion production in spallation reactions has been further investigated by performing dedicated Geant4 simulations of the $n_{-}$TOF spallation target. As suggested in Ref. 10, models producing overall more pions per incident proton also produce fewer neutrons, with a clear anticorrelation effect. On the contrary, prompt $\gamma$-rays, which are mostly produced by electromagnetic cascade following $\pi^{0}$ decay, correlate with the total number of pions in the hadronic cascade. This observation points out to a fundamental role of $\pi^{0}$ production in determining both the final neutron and $\gamma$-ray fluence produced by a spallation source based on high-energy protons on a heavy target, a phenomenon which is well known in the context of calorimetry detection in high-energy physics [14].

It has been further observed that, contrary to the thintarget case, the previous INCL version leads to thicktarget neutron and photon productions that are not sensibly different from the new version, except for very highenergy neutrons (above a few $\mathrm{GeV}$ ). This finding has been related to the structure of the whole hadronic cascade that develops in a thick spallation target; the emitted particles are shown to be sensitive only to the total number of pions produced along the hadronic cascade, in particular neutral pions. The mitigated dependence of thick-target yields on the underlying elementary cross sections is a direct consequence of conservation laws, and applies only for dimensions of the spallation target sensibly larger than the mean free path of secondary particles, as in the case of the $n$ TOF target considered in this work. This is valuable insight about the remarkable resilience of hadronic cascades and their manifest insensitivity to the details of the underlying physical models.

It remains to discuss to which extent secondary pions can be held responsible for the differences in neutron and photon yields predicted by the different Geant4 physics lists 10. While our work shows that pion-induced reactions can be relatively important, it should be borne in mind that other secondary particles are also at play. Secondary proton-nucleus reactions are seen to be less abundant than pion-nucleus reactions at these energies (see Fig. 10 and Table 1) and are probably better constrained in the models, due to the wide availability of elementary experimental data. This is much less the case for secondary neutron-nucleus reactions, which are sparsely represented in the elementary benchmarks of spallation models [e.g. 5-7, despite the fact that they are very common (Fig. 10 and Table 1). We believe that differences in the treatment of secondary neutrons and pions can account for most of the variation among the Geant4 physics lists.

This work was partially financed by the CHANDA EU FP7 project (grant agreement 605203). 


\section{References}

1. D. Filges and F. Goldenbaum. Handbook of spallation research. Theory, experiments and applications. Wiley-VCH, Berlin, 2009.

2. H. Aït Abderrahim, P. Baeten, D. De Bruyn, J. Heyse, P. Schuurmans, and J. Wagemans. MYRRHA, a Multipurpose hYbrid Research Reactor for High-end Applications. Nucl. Phys. News, 20(1):24-28, 2010. URL http://myrrha.sckcen.be/.

3. T. Enqvist, W. Wlazło, P. Armbruster, et al. Isotopic yields and kinetic energies of primary residues in $1 A$ $\mathrm{GeV}^{208} \mathrm{~Pb}+\mathrm{p}$ reactions. Nucl. Phys. A, 686(1-4):481524, April 2001. doi: 10.1016/S0375-9474(00)00563-7.

4. J.-C. David, A. Boudard, J. Cugnon, S. Ghali, S. Leray, D. Mancusi, and L. Zanini. Modeling astatine production in liquid lead-bismuth spallation targets. Eur. Phys. J. A, 49(3):29, March 2013. doi: 10.1140/epja/i2013-13029-4.

5. J-C. David, D. Filges, F. Gallmeier, M. Khandaker, A. Konobeyev, S. Leray, G. Mank, A. Mengoni, R. Michel, N. Otuka, and Y. Yariv. A new benchmark of spallation models. In Shielding Aspects of Accelerators, Targets and Irradiation Facilities - SATIF 10 Workshop Proceedings, page 273, Geneva, Switzerland, January 2011. OECD Publishing.

6. S. Leray, J.-C. David, M. Khandaker, G. Mank, A. Mengoni, N. Otsuka, D. Filges, F. Gallmeier, A. Konobeyev, and R. Michel. Results from the IAEA benchmark of spallation models. J. Korean Phys. Soc., 59(23):791-796, August 2011. doi: 10.3938/jkps. 59.791.

7. IAEA-intercomparison. IAEA benchmark of spallation models. http://www-nds.iaea.org/ spallations, 2009.

8. C. Guerrero, A. Tsinganis, E. Berthoumieux, et al. Performance of the neutron time-of-flight facility $\mathrm{n}$ TOF at CERN. Eur. Phys. J. A, 49(2):27, February 2013.

9. Barbagallo, M., Guerrero, C., Tsinganis, A., et al. High-accuracy determination of the neutron flux at n_TOF. Eur. Phys. J. A, 49(12):156, December 2013. doi: $10.1140 /$ epja/i2013-13156-x.

10. S. Lo Meo, M. A. Cortés-Giraldo, C. Massimi, et al. GEANT4 simulations of the n_TOF spallation source and their benchmarking. Eur. Phys. J. A, 51(12):160, December 2015.

11. J. Lerendegui, S. Lo Meo, C. Guerrero, et al. Geant4 simulation of the $\mathrm{n}$ _TOF-EAR2 neutron beam: characteristics and prospects. Eur. Phys. J. A, 52(4):100, 2016. doi: $10.1140 /$ epja/i2016-16100-8.

12. S. Agostinelli et al. GEANT4 - a simulation toolkit. Nucl. Instrum. Meth. A, 506(3):250-303, July 2003. Official web site: http://geant4.cern.ch/

13. J. Allison et al. GEANT4 developments and applications. IEEE T. Nucl. Sci., 53(1):270-278, February 2006.

14. C. W. Fabjan and F. Gianotti. Calorimetry for particle physics. Rev. Mod. Phys., 75:1243-1286, October 2003. doi: 10.1103/RevModPhys.75.1243.
15. A. Boudard, J. Cugnon, S. Leray, and C. Volant. Intranuclear cascade model for a comprehensive description of spallation reaction data. Phys. Rev. C, 66 (4):044615, October 2002. doi: 10.1103/PhysRevC. 66.044615 .

16. D. Mancusi, A. Boudard, J. Cugnon, J.-C. David, P. Kaitaniemi, and S. Leray. Extension of the Liège intranuclear-cascade model to reactions induced by light nuclei. Phys. Rev. C, 90:054602, November 2014. doi: 10.1103/PhysRevC.90.054602.

17. J. M. Quesada, V. Ivanchenko, A. Ivanchenko, M. A. Cortés-Giraldo, G. Folger, A. Howard, and D. Wright. Recent developments in pre-equilibrium and deexcitation models in Geant4. Prog. Nucl. Sci. Technol., 2:936-941, October 2011.

18. J.-J. Gaimard and K.-H. Schmidt. A reexamination of the abrasion-ablation model for the description of the nuclear fragmentation reaction. Nucl. Phys. A, 531(3-4):709-745, September 1991.

19. A.R. Junghans, M. de Jong, H.-G. Clerc, A.V. Ignatyuk, G.A. Kudyaev, and K.-H. Schmidt. Projectile-fragment yields as a probe for the collective enhancement in the nuclear level density. Nucl. Phys. A, 629(3-4):635-655, February 1998. doi: 10. 1016/S0375-9474(98)00658-7.

20. A. Kelić, M. V. Ricciardi, and K.-H. Schmidt. ABLA07 - towards a complete description of the decay channels of a nuclear system from spontaneous fission to multifragmentation. In Joint ICTP-IAEA Advanced Workshop on Model Codes for Spallation Reactions, page 181, Trieste, Italy, 2008. IAEA. Report INDC(NDC)-0530.

21. Th. Aoust and J. Cugnon. Pion physics in the Liège intranuclear cascade model. Phys. Rev. C, 74:064607, December 2006. doi: 10.1103/PhysRevC.74.064607.

22. Th. Aoust and J. Cugnon. Effects of isospin and energy dependences of the nuclear mean field in spallation reactions. Eur. Phys. J. A, 21(1):79-85, 2004. doi: 10.1140/epja/i2003-10186-y.

23. K. Ackerstaff, J. Bisplinghoff, R. Bollmann, et al. A hadron-nucleus collision event generator for simulations at intermediate energies. Nucl. Instrum. Meth. A, 491(3):492-506, 2002. doi: 10.1016/S0168-9002(02) 01240-8.

24. V. Flaminio, W. G. Moorhead, D. R. O. Morrison, and N. Rivoire. Compilation of cross-sections $I: \pi^{+}$ and $\pi^{-}$induced reactions. CERN, Geneva, 1983. URL https://cds.cern.ch/record/99267.

25. V. Flaminio, W. G. Moorhead, D. R. O. Morrison, and N. Rivoire. Compilation of cross-sections III: $p$ and $\bar{p}$ induced reactions. CERN, Geneva, 1984. URL https://cds.cern.ch/record/101631.

26. S. Pedoux and J. Cugnon. Extension of the Liège intranuclear cascade model at incident energies between 2 and $12 \mathrm{GeV}$. Aspects of pion production. Nucl. Phys. A, 866(1):16-36, September 2011.

27. S. Pedoux. Extension of the Liège intranuclear cascade model to the 2-15 GeV incident energy range. $\mathrm{PhD}$ thesis, University of Liège, Liège, Belgium, 2011. 
28. G. Folger, V.N. Ivanchenko, and J.P. Wellisch. The Binary Cascade. Eur. Phys. J. A, 21:407-417, 2004. ISSN 1434-6001. doi: 10.1140/epja/i2003-10219-7.

29. D.H. Wright and M.H. Kelsey. The Geant4 Bertini cascade. Nucl. Instrum. Meth. A, 804:175-188, 2015. doi: 10.1016/j.nima.2015.09.058.

30. M. G. Catanesi, E. Radicioni, R. Edgecock, et al. Large-angle production of charged pions with 3-12.9 $\mathrm{GeV} / c$ incident protons on nuclear targets. Phys. Rev. C, 77:055207, May 2008. doi: 10.1103/PhysRevC.77. 055207.

31. M. Apollonio, A. Artamonov, A. Bagulya, et al. Large-angle production of charged pions with incident pion beams on nuclear targets. Phys. Rev. C, 80:065207, December 2009. doi: 10.1103/PhysRevC. 80.065207 .

32. A. Boudard, J. Cugnon, J.-C. David, S. Leray, and D. Mancusi. New potentialities of the Liège intranuclear cascade (INCL) model for reactions induced by nucleons and light charged particles. Phys. Rev. C, 87 (1):014606, January 2013.

33. E. Oset and L.L. Salcedo. Delta self-energy in nuclear matter. Nucl. Phys. A, 468(3):631-652, 1987. doi: 10.1016/0375-9474(87)90185-0.

34. B. ter Haar and R. Malfliet. Nucleons, mesons and deltas in nuclear matter a relativistic Dirac-Brueckner approach. Phys. Rep., 149(4):207-286, 1987. doi: 10. 1016/0370-1573(87)90085-8.

35. Y. Yariv and Z. Fraenkel. Intranuclear cascade calculation of high-energy heavy-ion interactions. Phys. Rev. C, 20(6):2227-2243, December 1979.

36. Y. Yariv and Z. Fraenkel. Intranuclear cascade calculation of high energy heavy ion collisions: Effect of interactions between cascade particles. Phys. Rev. C, 24(2):488-494, August 1981.

37. Harp-cdp website. http://harp-cdp.web.cern.ch/

38. A. Bolshakova, I. Boyko, G. Chelkov, et al. Crosssections of large-angle hadron production in protonand pion-nucleus interactions $\mathrm{V}$ : lead nuclei and beam momenta from $\pm 3 \mathrm{GeV} / c$ to $\pm 15 \mathrm{GeV} / c$. Eur. Phys. J. C, 66(1-2):57-117, 2010. doi: 10.1140/epjc/ s10052-010-1249-y.

39. I. Chemakin, V. Cianciolo, B. A. Cole, et al. Inclusive soft pion production from 12.3 and $17.5 \mathrm{GeV} / c$ protons on $\mathrm{Be}, \mathrm{Cu}$, and $\mathrm{Au}$. Phys. Rev. C, 65:024904, January 2002. doi: 10.1103/PhysRevC.65.024904.

40. J. Apostolakis, G. Folger, V. Grichine, et al. Progress in hadronic physics modelling in Geant4. J. Phys.: Conf. Ser., 160(1):012073, 2009.

41. H. Schneuwly. Exotic atoms. In G. Fiorentini and G. Torelli, editors, Proc. of the 1st Course of the Int. School of Physics of Exotic Atoms, Erice, Italy, April 1977.

42. T. Koi. Shielding benchmarks for Geant 4 version 10. In Proceedings of the Twelfth Workshop on Shielding Aspects of Accelerators, Targets and Irradiation Facilities - SATIF 12, pages 269-275, Batavia, IL, U.S.A., April 2014. Nuclear Energy Agency. 